\title{
Oxidative Stress and its Different Roles in Neurodegenerative Diseases
}

\section{Sadegh Rajabi ${ }^{1,2}$, Shokoofeh Noori², Fatemeh Zal ${ }^{3}$, Ali Jahanbazi Jahan-Abad ${ }^{1 \text {,* }}$}

${ }^{1}$ Shefa Neuroscience Research Center, Khatam Alanbia Hospital, Tehran, Iran

${ }^{2}$ Department of Clinical Biochemistry, Faculty of Medicine, Shahid Beheshti University of Medical Sciences, Tehran, Iran

${ }^{3}$ Reproductive Biology Department, School of Advanced Medical Sciences and Technologies, Shiraz University of Medical Sciences, Shiraz, Iran

\section{ABSTRACT}

Introduction: The incidence and prevalence of neurodegenerative diseases increase with life expectancy. Brain, for physiological and biochemical reasons, has a high sensitivity to oxidative stress. Therefore, maintaining the redox homeostasis is essential for brain cells. In addition, brain antioxidant levels are limited compared to other tissues. In this article, different mechanisms involved in the production of endogenous and exogenous reactive oxygen species and the role of oxidative stress in neurodegenerative diseases were discussed. Redox imbalance occurs when antioxidant capacity doesn't overcome free radicals, which can lead to tissue damage, cell death or disease onset. This article also reviews various molecular and signaling mechanisms involved in oxidative stress management in neurodegenerative diseases.

Conclusion: Although the induction and role of oxidative stress in neurodegenerative disease have been approved, its role in pathogenesis of some of the neurodegenerative diseases needs to be further investigated. It is possible that with antioxidant therapy, we could modulate oxidative status and prevent or treat these diseases.

\section{Key words:}

1. Neurodegenerative

Diseases

2. Reactive Oxygen Species

3. Antioxidants

*Corresponding Author: Ali Jahanbazi Jahan-Abad

E-mail:a.jahanbazi65@yahoo.com

doi: 10.18869/acadpub.shefa.5.1.73 
استرس اكسيداتيو و نقشهاى مختلف آن در بيمارىهاى تحليل برندهُ عصبى

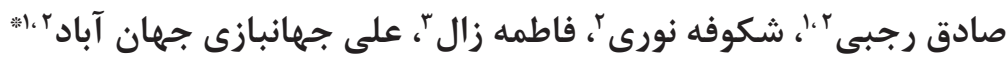

'مركز تحقيقات علوم اعصاب شفا، بيمارستان خاتمالانبياء، تهران، ايران

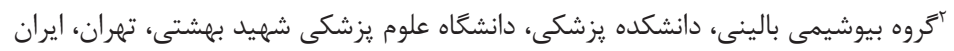

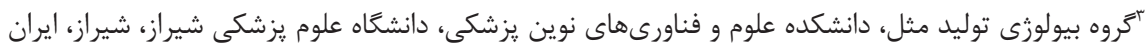

كليد وازهها:

1. بيمارىهـاى تحليـل

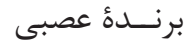

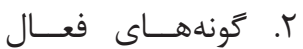

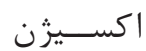

با

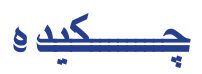

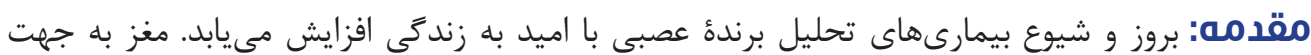

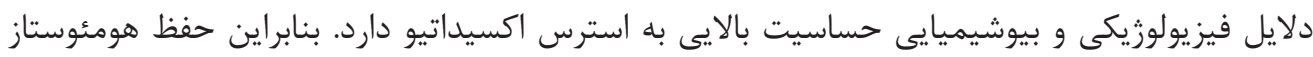

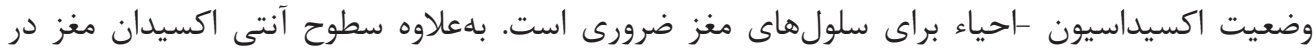

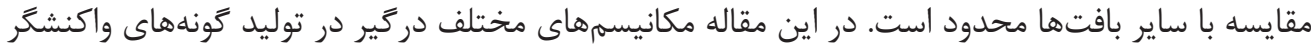

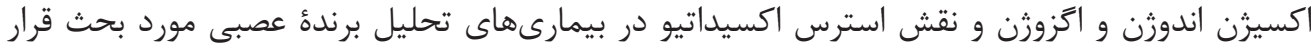

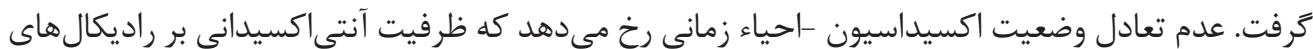

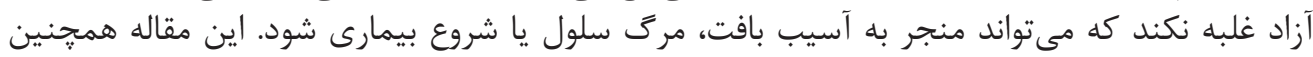

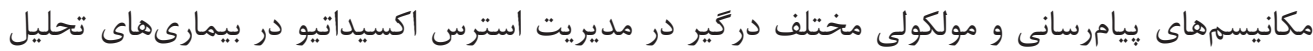

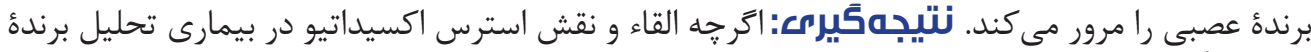

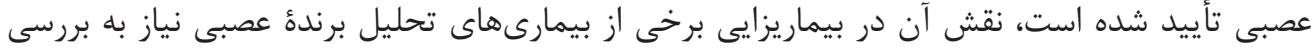

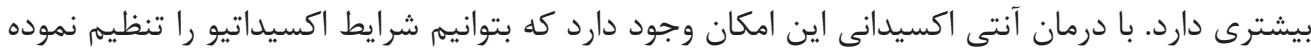

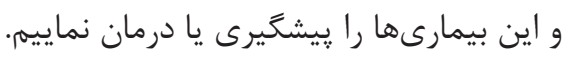

" نويسنده مسئول: على جهانبازى جهان آباد a.jahanbazi65@yahoo.com آدرس الكترونيكى 


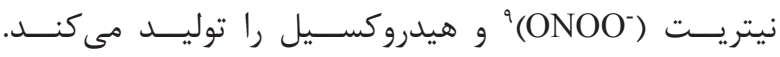

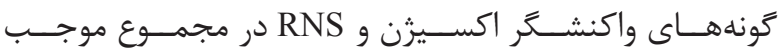

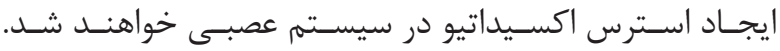

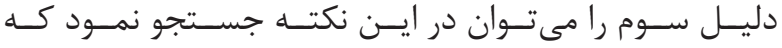

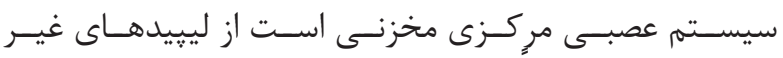

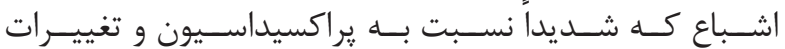

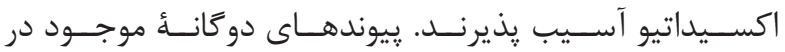

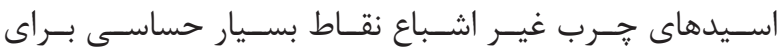

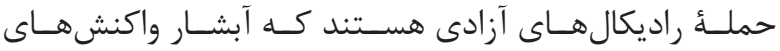

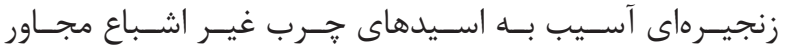

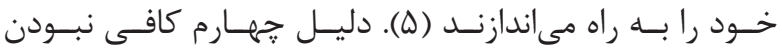

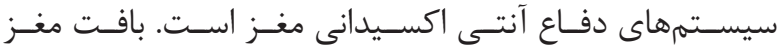

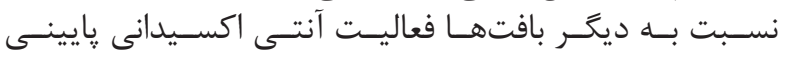

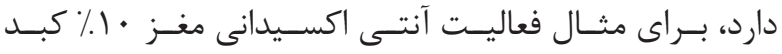

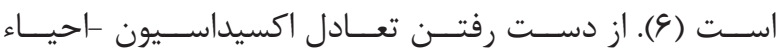

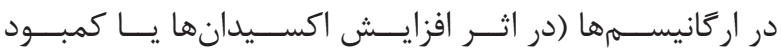

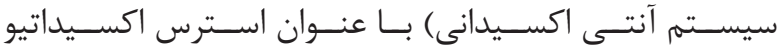

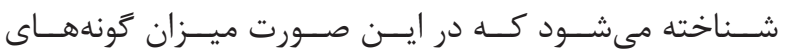

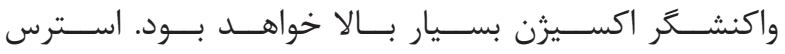

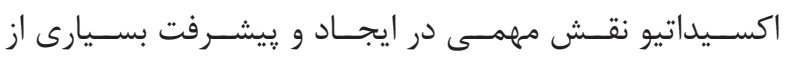

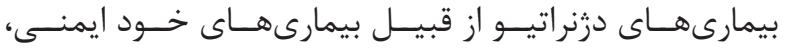

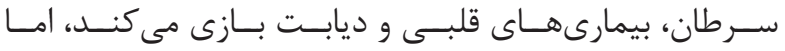

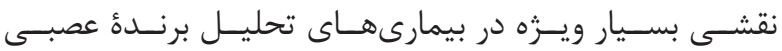

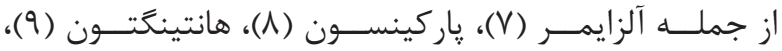

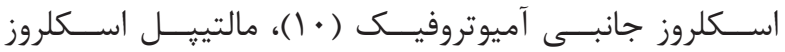

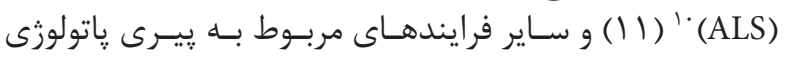

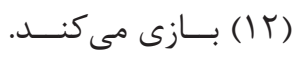

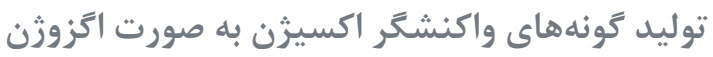

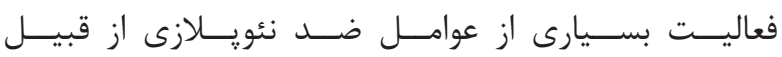

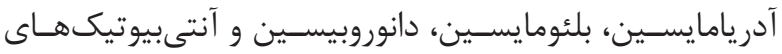

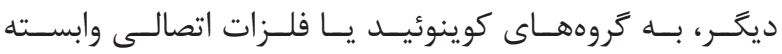

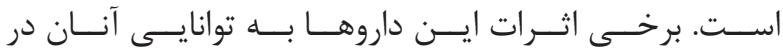

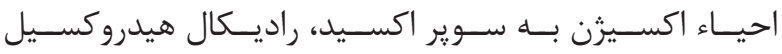

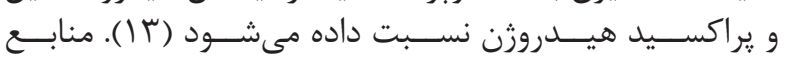

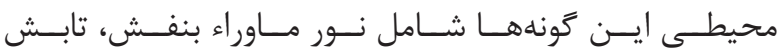

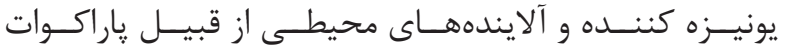

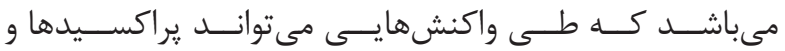

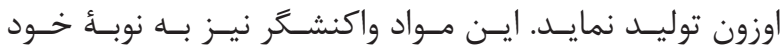

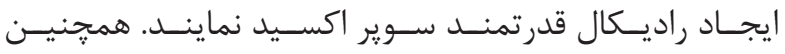

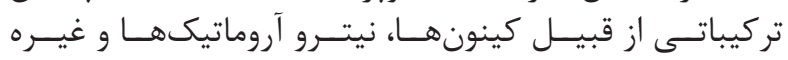

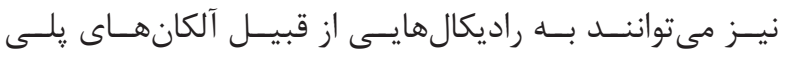

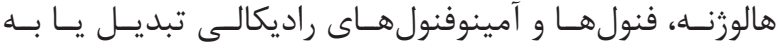

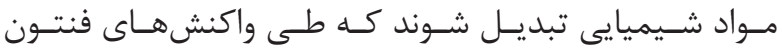

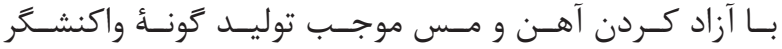

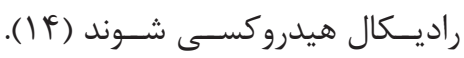

${ }^{1}$ Neurodegenerative

${ }^{2}$ Oxidative stress

${ }^{3}$ Excitotoxicity

${ }^{4}$ Reactive oxygen species

${ }^{5}$ Central nervous system

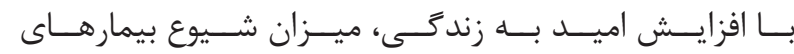

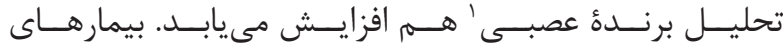

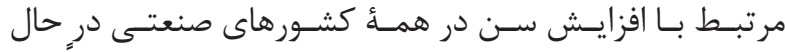

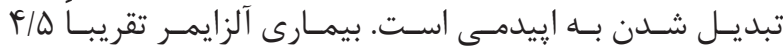

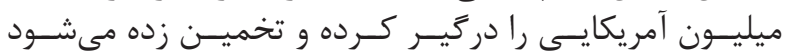

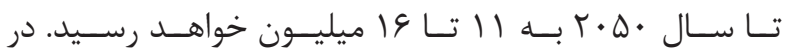

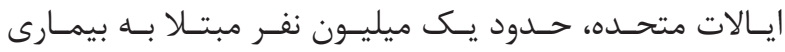

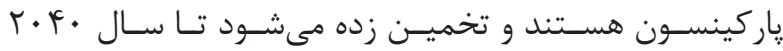

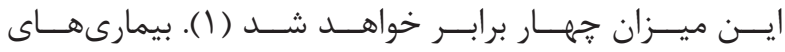

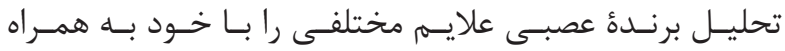

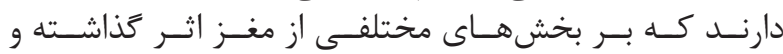

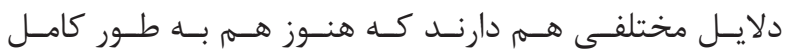

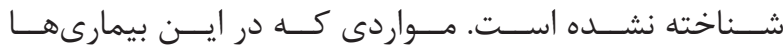

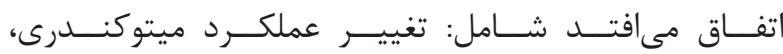

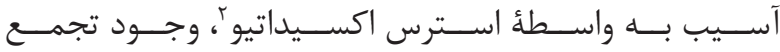

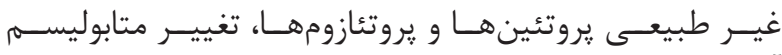

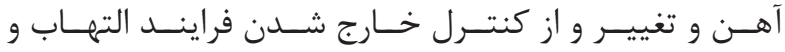

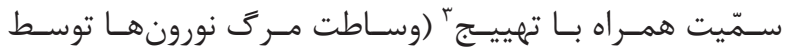

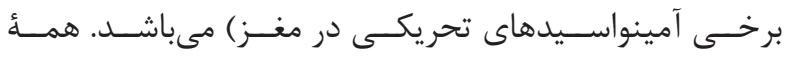

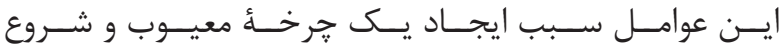

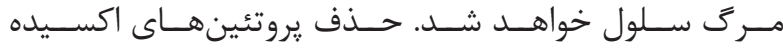

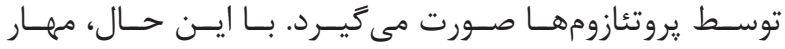

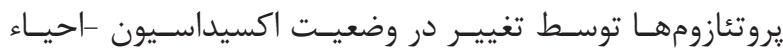

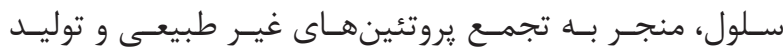

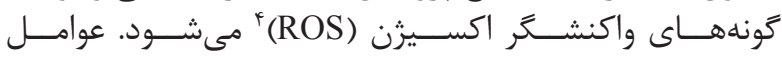

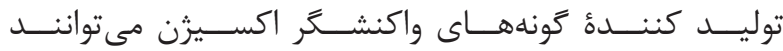

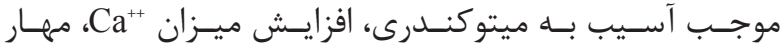

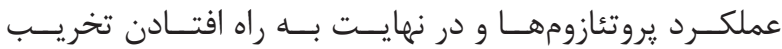

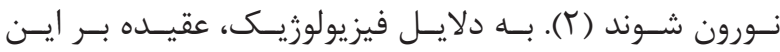

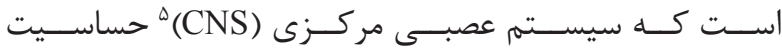

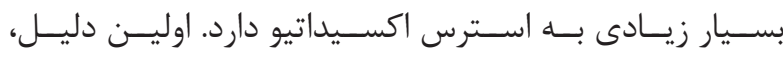

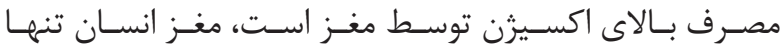

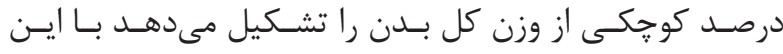

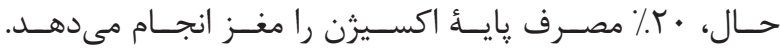

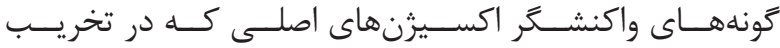

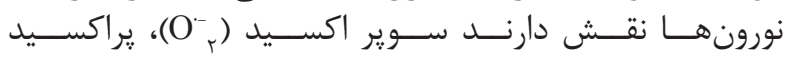

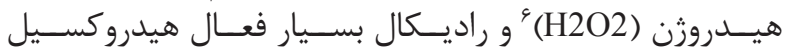

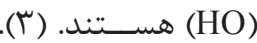

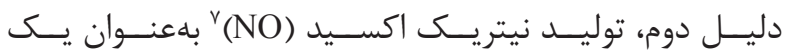

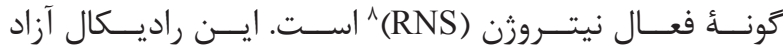

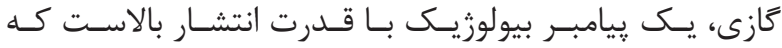

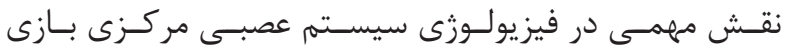

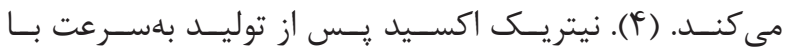

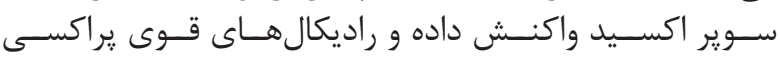

${ }^{6}$ Hydrogen peroxide

${ }^{7}$ Nitric oxide

${ }^{8}$ Reactive nitrogen species

${ }^{9}$ Peroxynitrite

${ }^{10}$ Amyotrophic lateral sclerosis 


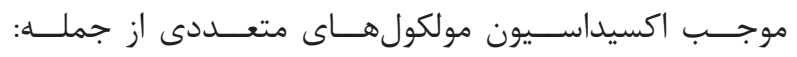

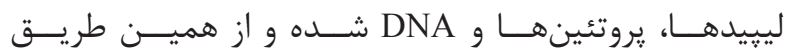

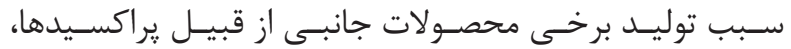

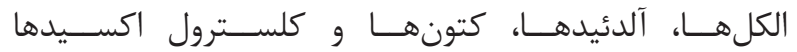

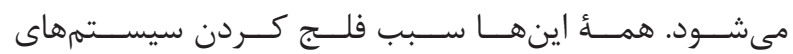

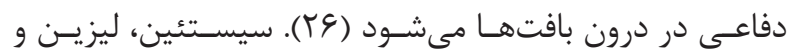

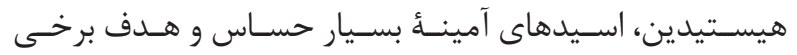

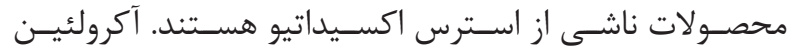

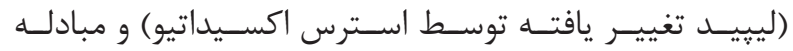

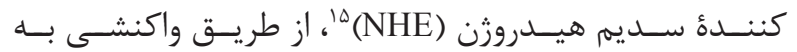

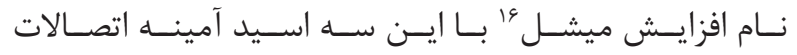
عرضــى برقــرار مى كنـــــد (YV)

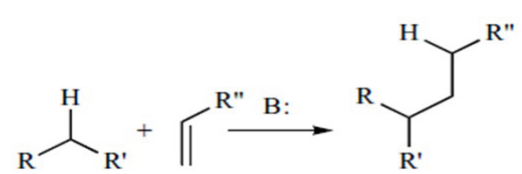

Where $\mathrm{B}$ is the Base e.g. $\mathrm{NaOH}, \mathrm{KOH}$ etc.

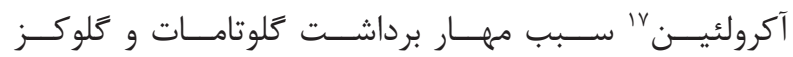

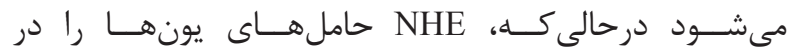

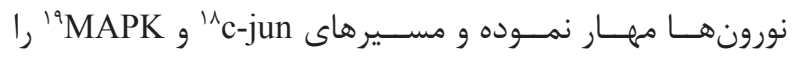

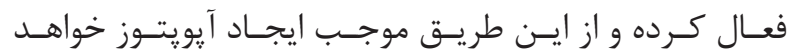

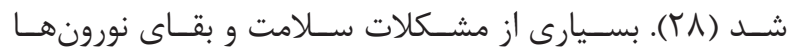

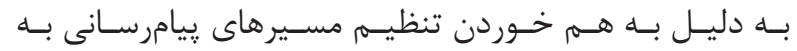

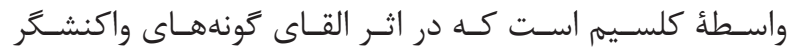

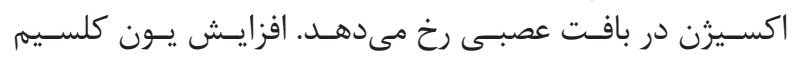

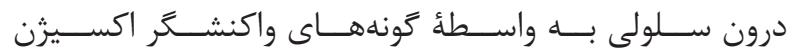

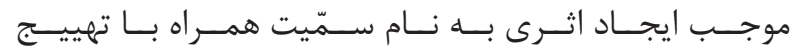

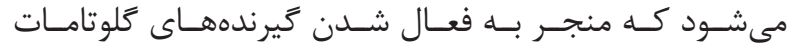

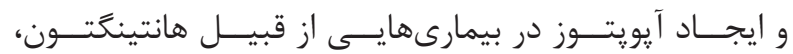

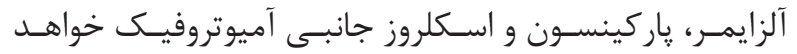

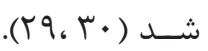

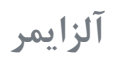

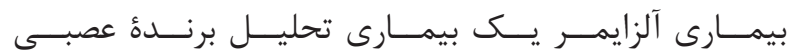

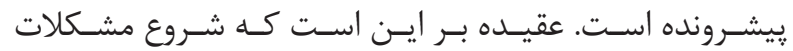

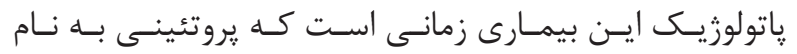

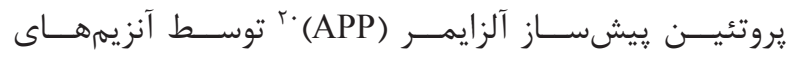

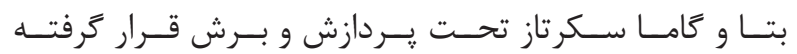

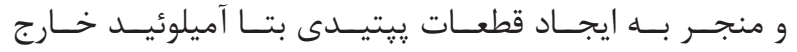

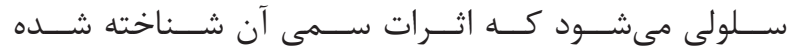

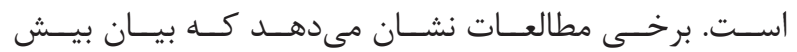

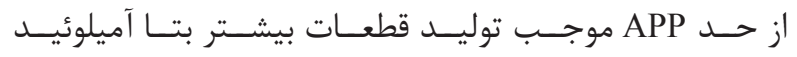

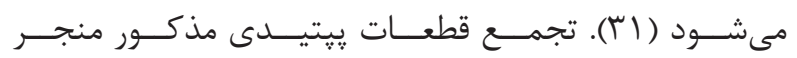

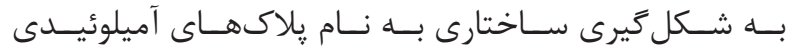

${ }^{11}$ Monoamine oxidase

${ }^{12}$ Neurotransmitters

${ }^{13}$ 1-Methyl-4-phenyl-1,2,3,6-tetrahydropyridine

${ }^{14}$ Nitric oxide synthase, inducible

${ }^{15}$ Sodium-hydrogen exchanger

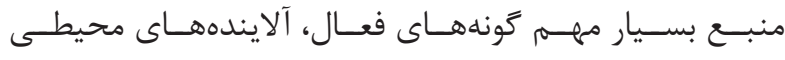

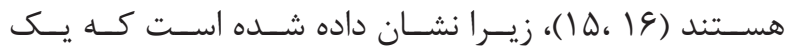

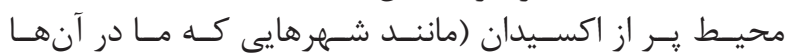

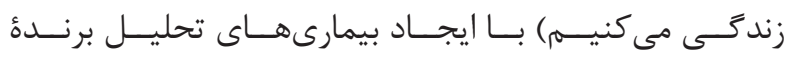

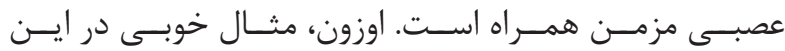

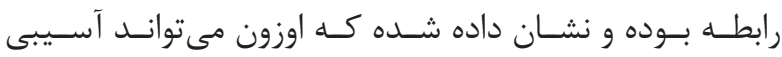

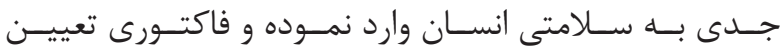

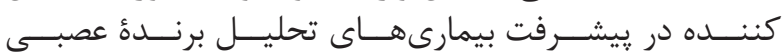

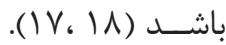

توليد كونههاى واكنشعَ اكسيثزن به صورت اندوزن

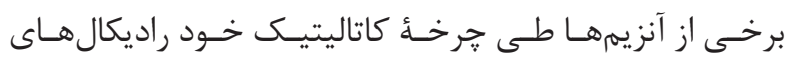

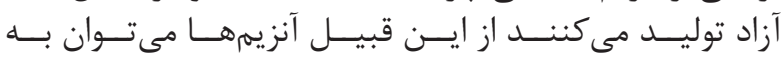

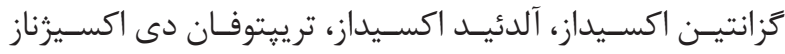

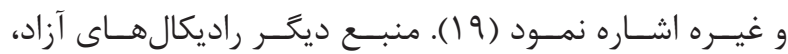

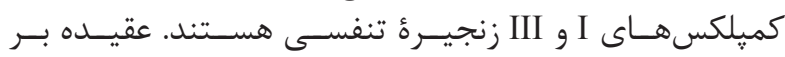

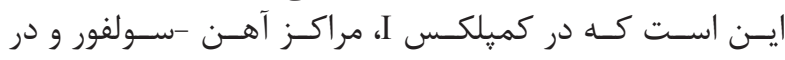

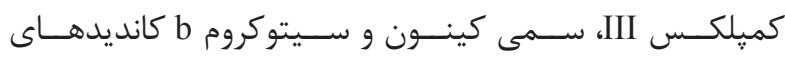

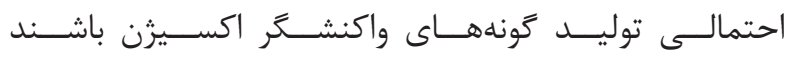

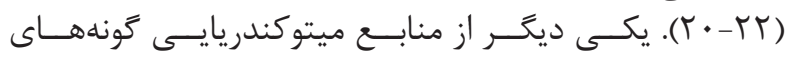

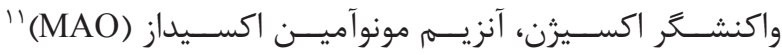

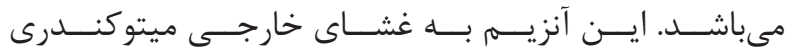

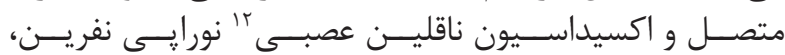

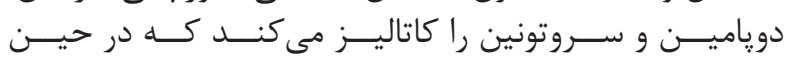

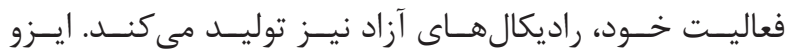

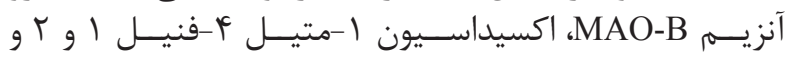

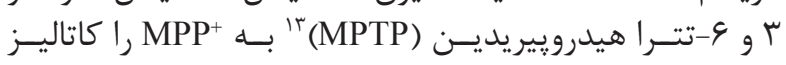

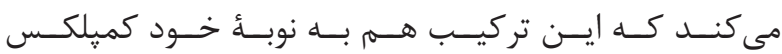

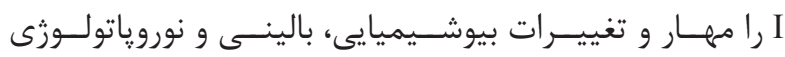

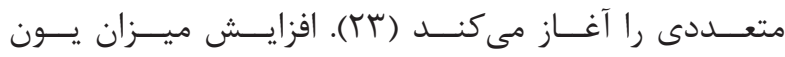

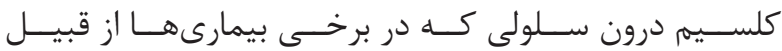

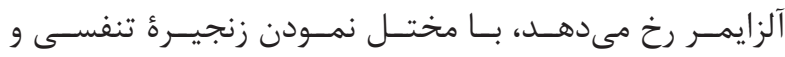

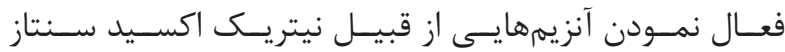
(iNOS)

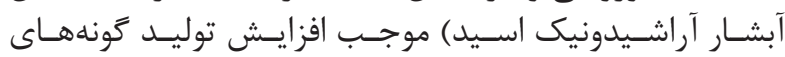

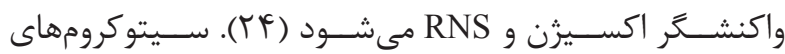

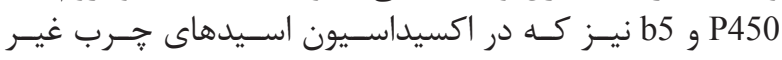

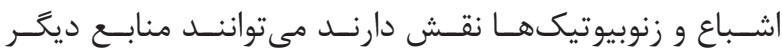

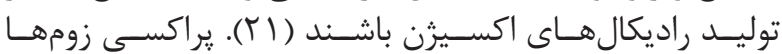

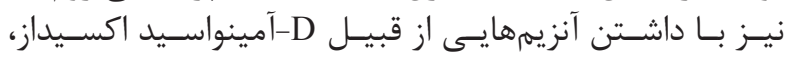

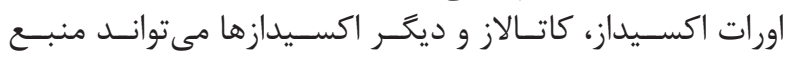

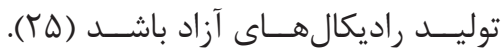

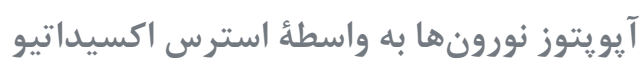

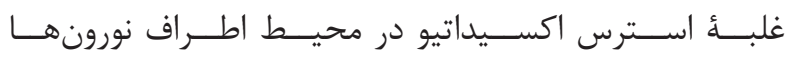

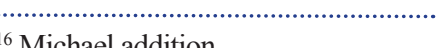

${ }^{16}$ Michael addition

${ }^{17}$ Acrolein

${ }^{18}$ Jun proto-oncogene

${ }^{19}$ Mitogen-activated protein kinases

${ }^{20}$ Amyloid precursor protein 
نهايـت منجــر بــه مــرى سـلولى نكــروزى مىشـود (بَ).

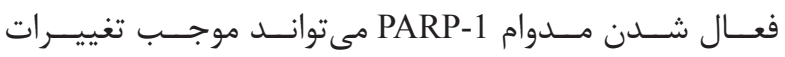

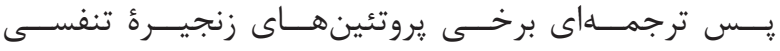

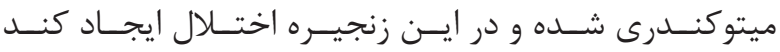

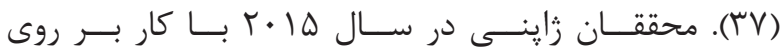

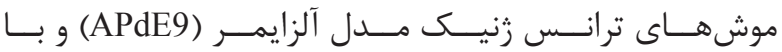

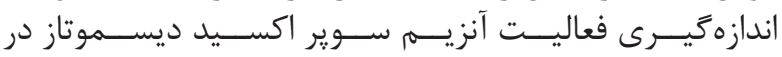

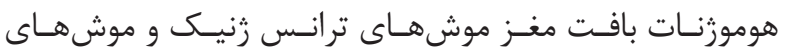

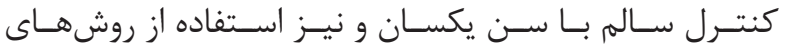

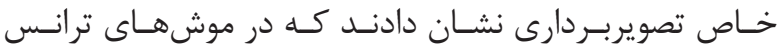

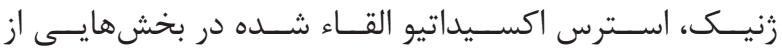

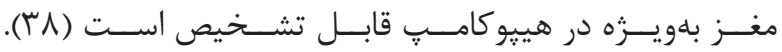

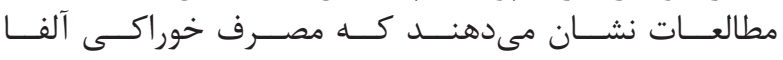

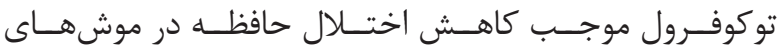

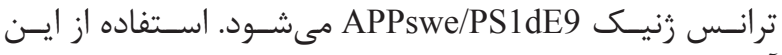

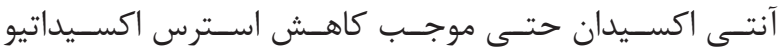

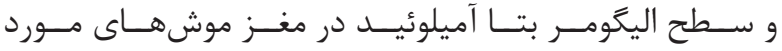

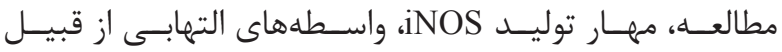

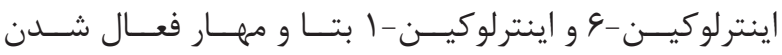

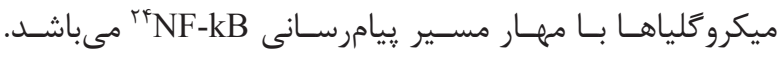

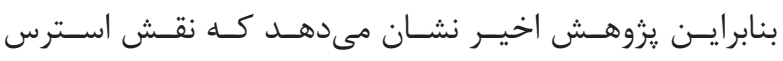

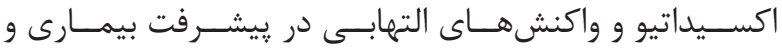

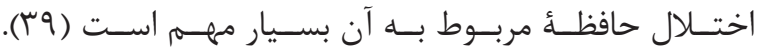

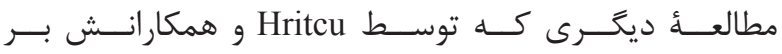

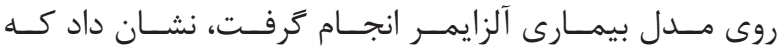

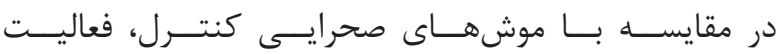

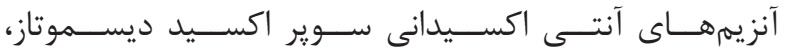

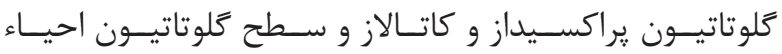

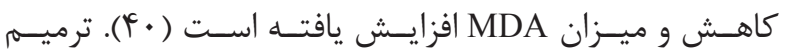

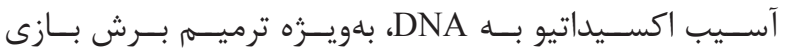

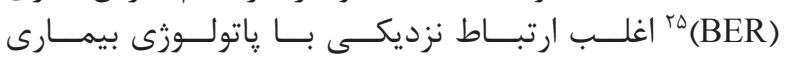

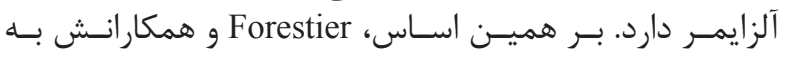

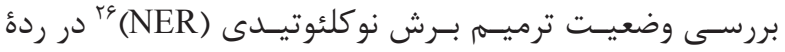

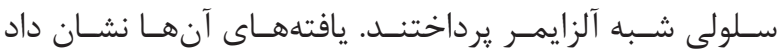

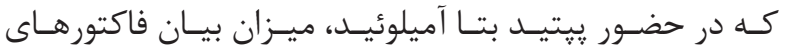

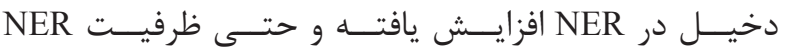

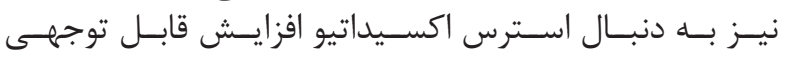

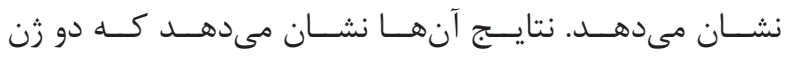

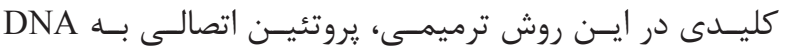

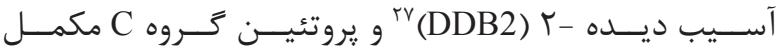

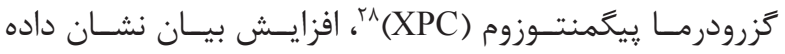

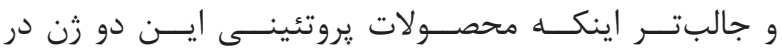

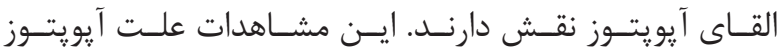

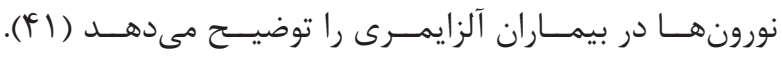

${ }^{21}$ Malondialdehyde

22 4-Hydroxynonenal

${ }^{23}$ Poly [ADP-ribose] polymerase 1

${ }^{24}$ Nuclear factor kappa-light-chain-enhancer of activated B cells

${ }^{25}$ Base excision repair

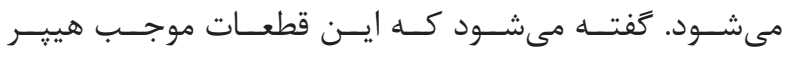

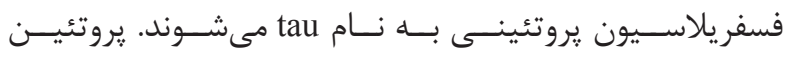

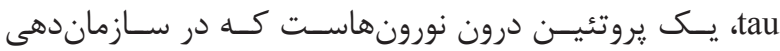

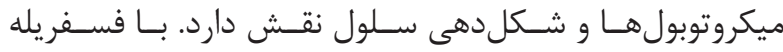

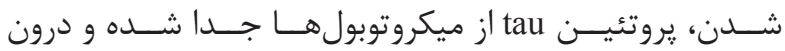

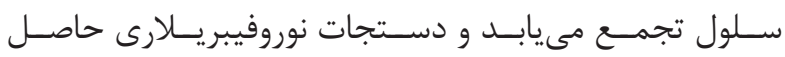

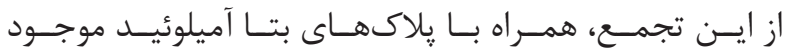

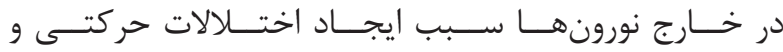

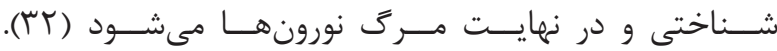

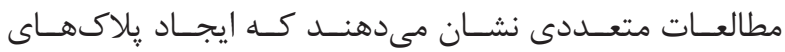

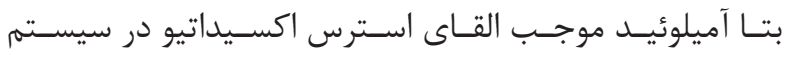

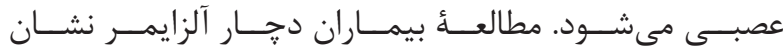

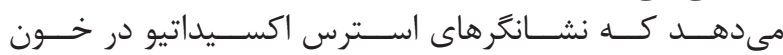

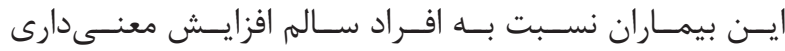

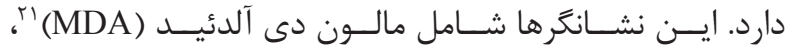

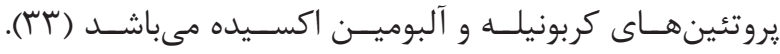
و و همكارانـش نشـان دادنـد Sultana

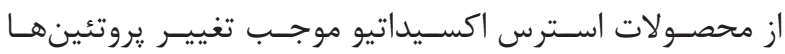

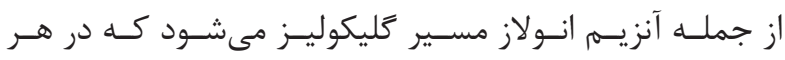

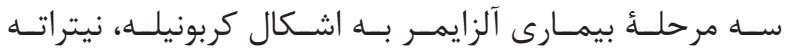

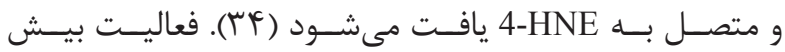

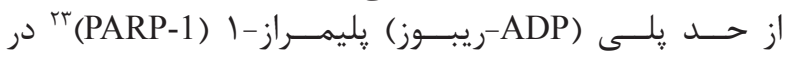

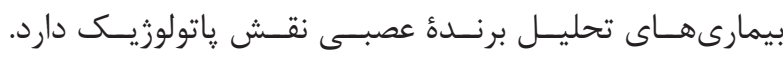

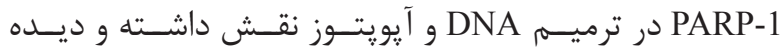

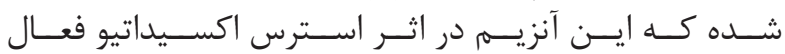

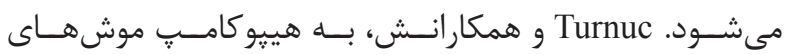

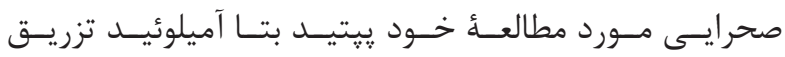

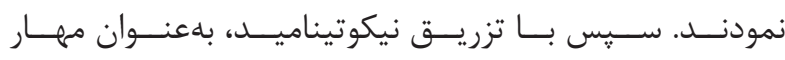
كنـــــده MDA

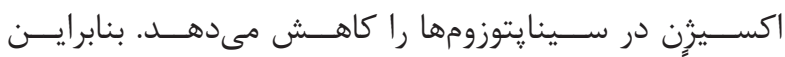

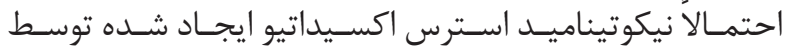

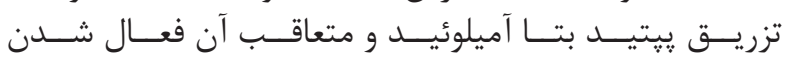

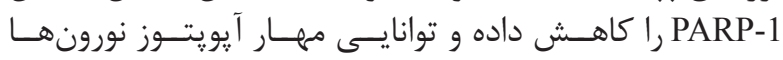

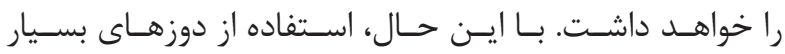

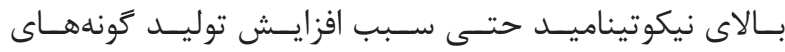

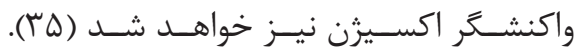

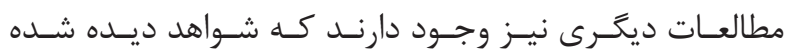

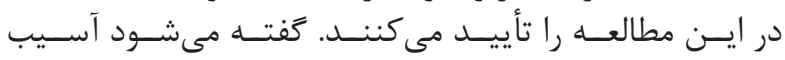

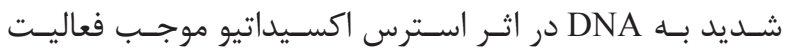

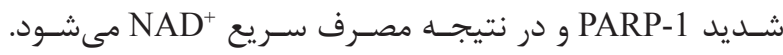

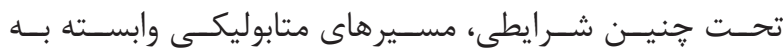
NAD+

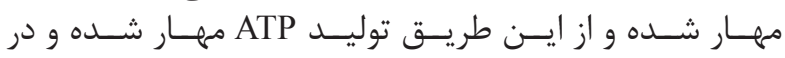

\footnotetext{
${ }^{26}$ Nucleotide excision repair

${ }^{27}$ DNA binding protein 2

${ }^{28}$ Xeroderma pigmentosum, complementation group C

${ }^{29}$ Nuclear factor-erythroid 2-related factor 2
} 


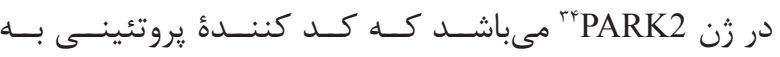

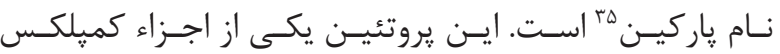

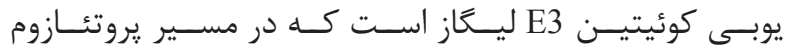

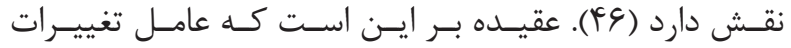

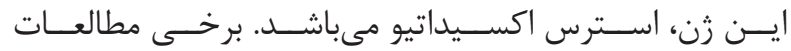

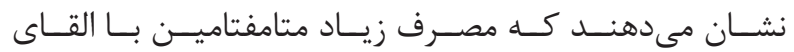

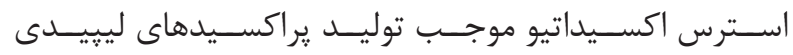

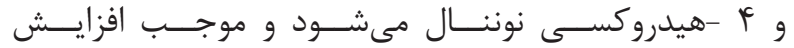

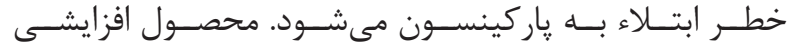

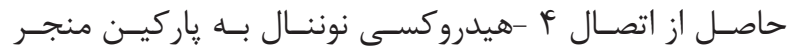

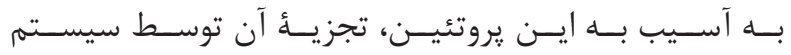

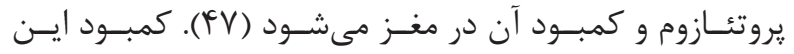

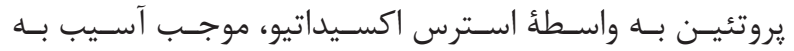

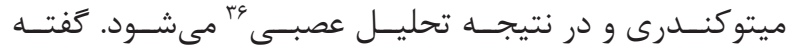

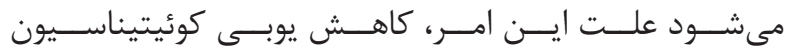

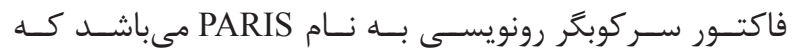

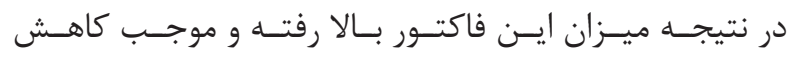

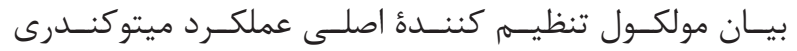

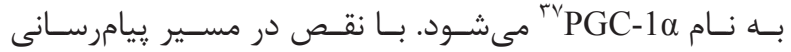

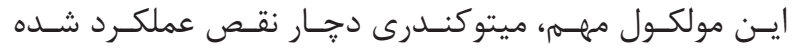

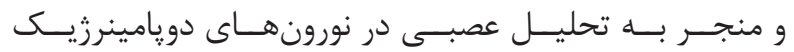

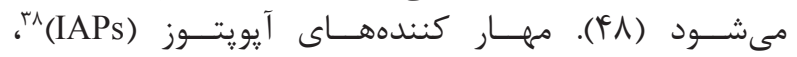

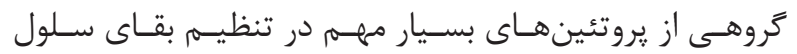

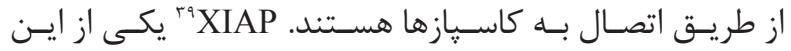

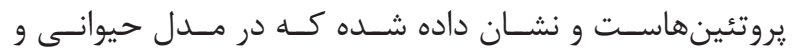

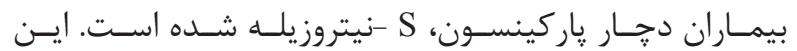

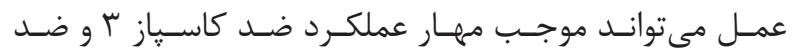

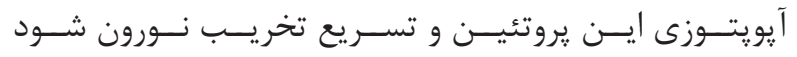

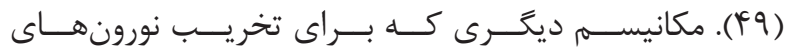

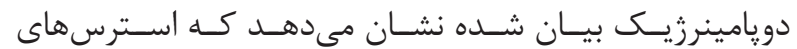

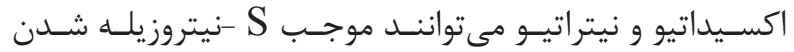

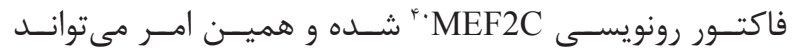

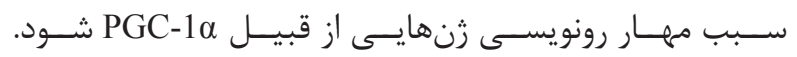

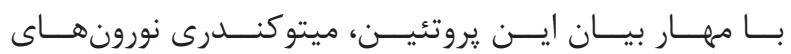

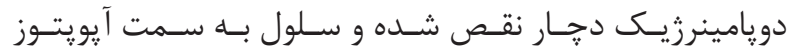

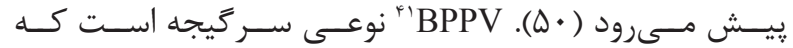

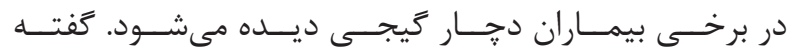

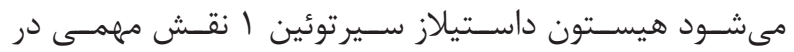

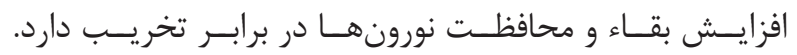

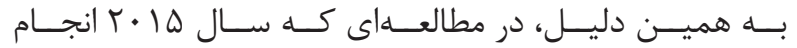

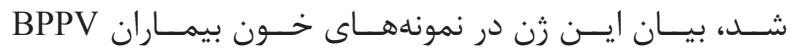

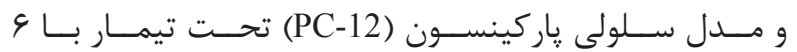

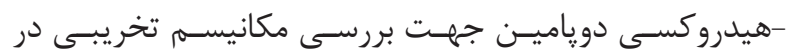

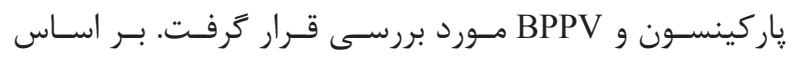

\footnotetext{
${ }^{30}$ Superoxide dismutase 1

${ }^{31}$ Pathogenicity

${ }^{32}$ Beta amyloid 1-42

${ }^{33}$ A disintegrin and metalloproteinase domain-containing protein 10

${ }^{34}$ Parkin RBR E3 ubiquitin protein ligase

${ }^{35}$ Parkin
}

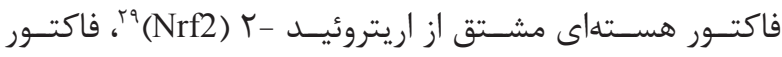

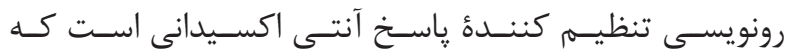

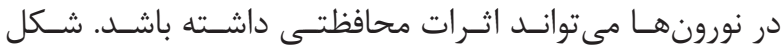

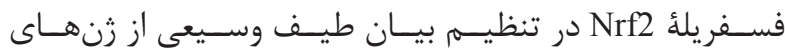

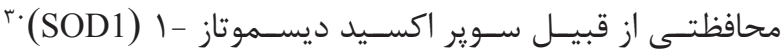

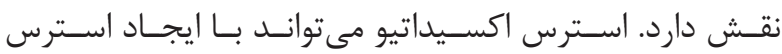

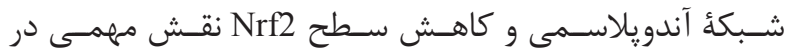

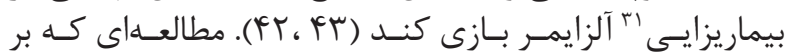

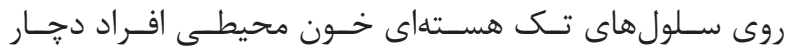

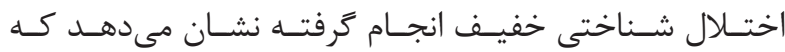

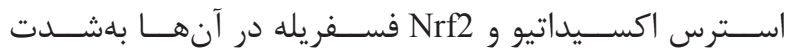

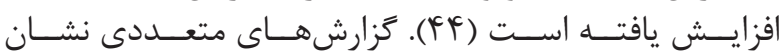

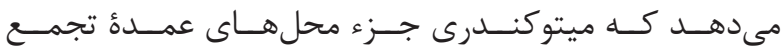

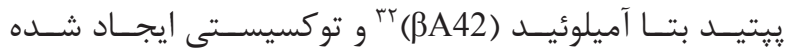

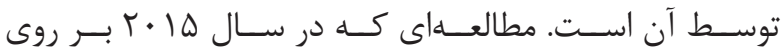

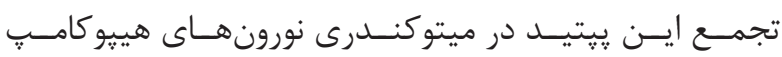

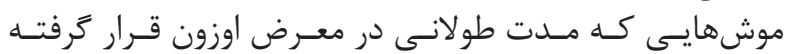

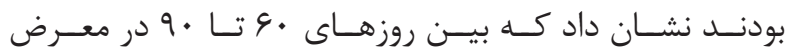

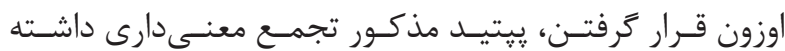

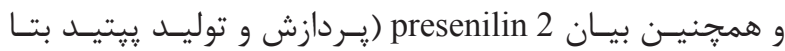

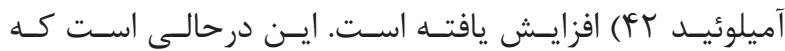

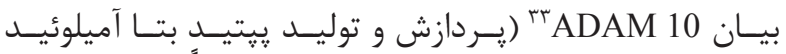

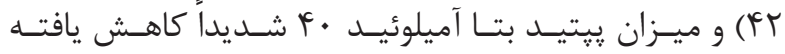

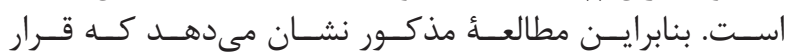

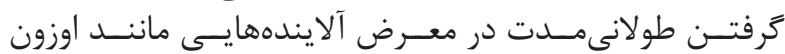

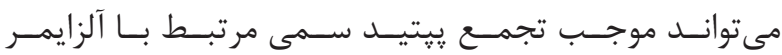

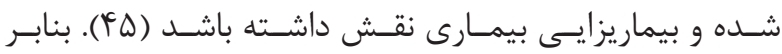

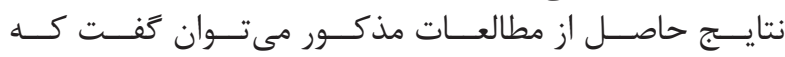

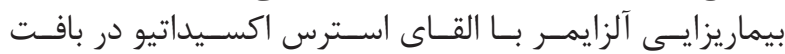

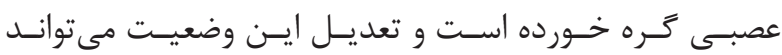

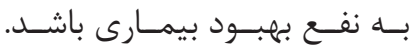

ياركينسون

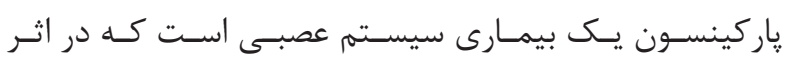

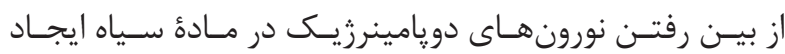

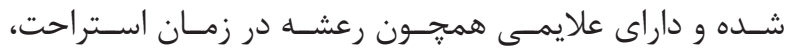

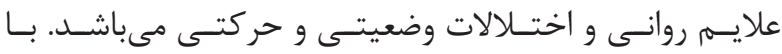

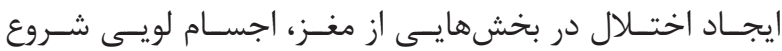

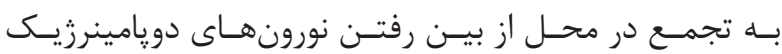

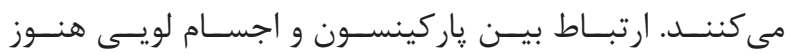

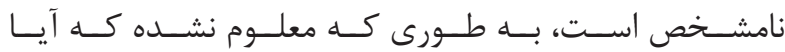

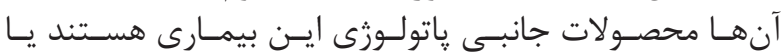

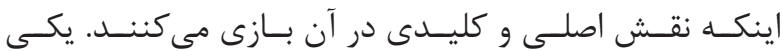

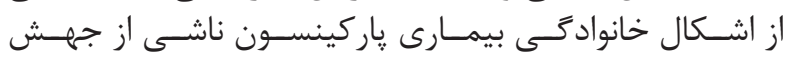

\footnotetext{
${ }^{36}$ Neurodegeneration

${ }^{37}$ Peroxisome proliferator-activated receptor gamma coactivator 1-alpha

${ }^{38}$ Inhibitors of apoptosis proteins

${ }^{39} \mathrm{X}$-linked IAP

${ }^{40}$ Myocyte enhancer factor $2 \mathrm{c}$

${ }^{41}$ Benign paroximal positional vertigo
} 


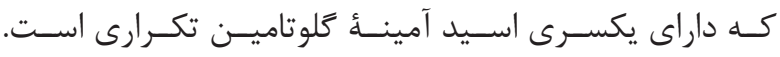

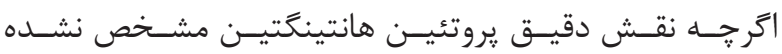

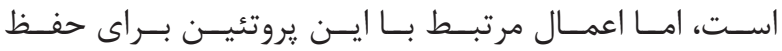

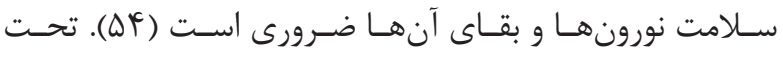

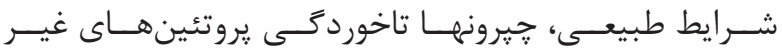

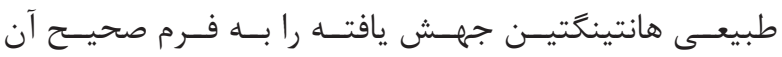

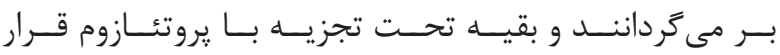

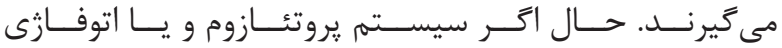

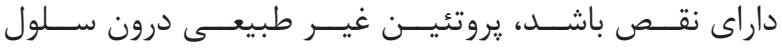

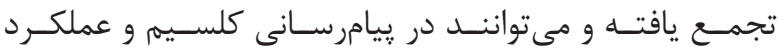

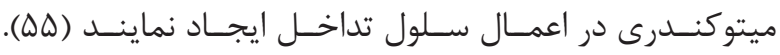

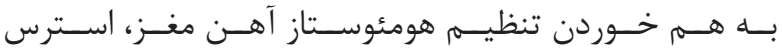

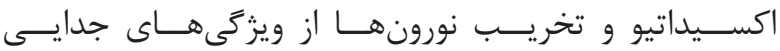

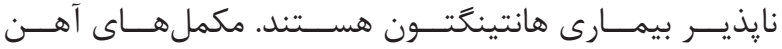

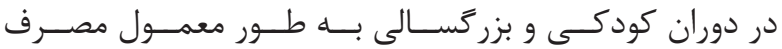

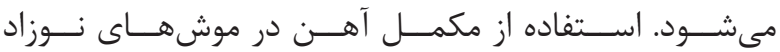

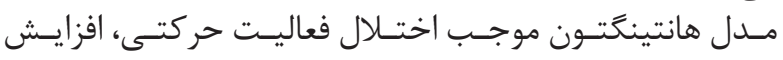

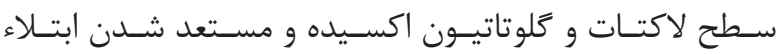

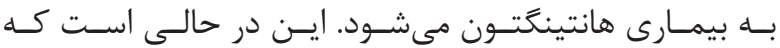

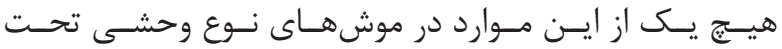

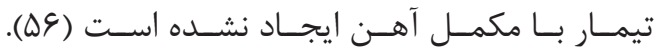

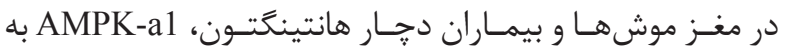

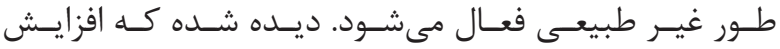

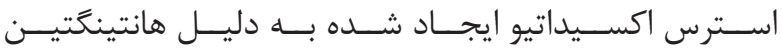

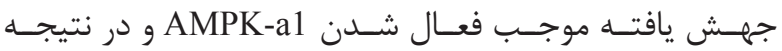

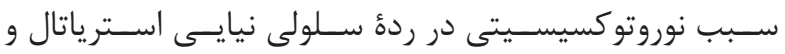

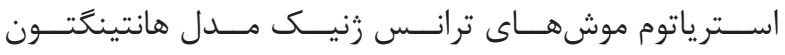

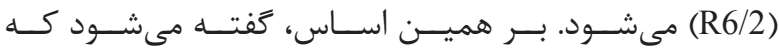

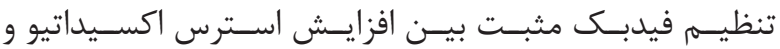

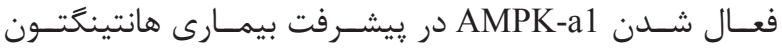

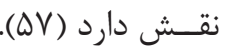

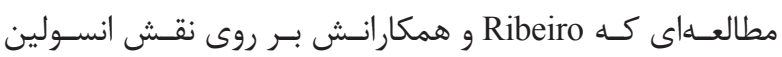

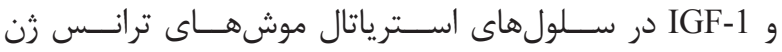

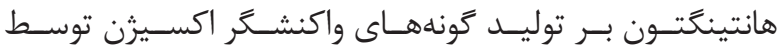

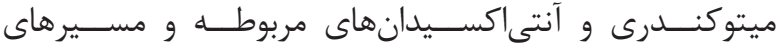

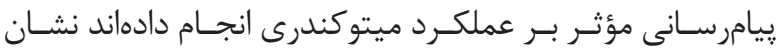

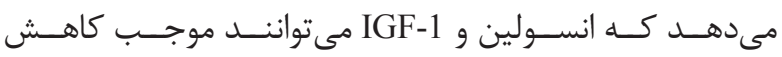

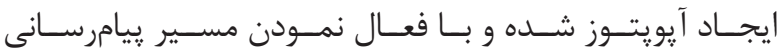

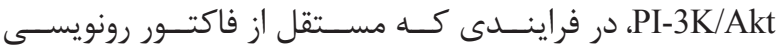

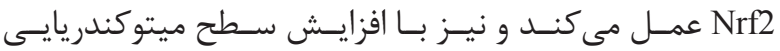

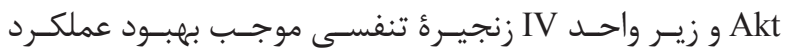

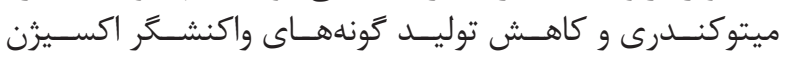

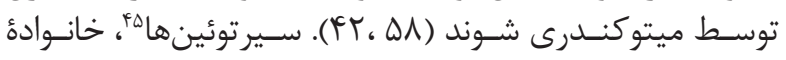

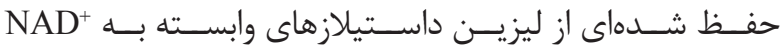

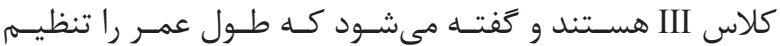

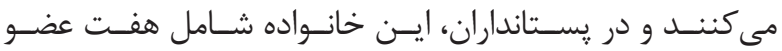

${ }^{42}$ PTEN-induced putative kinase 1

${ }^{43}$ Huntingtin gene

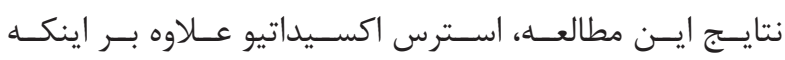

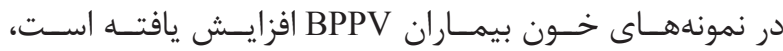

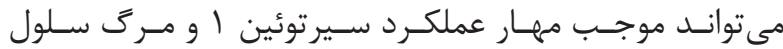

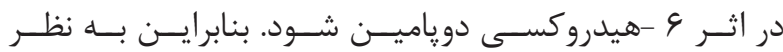

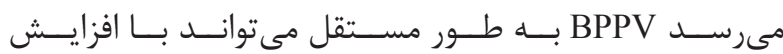

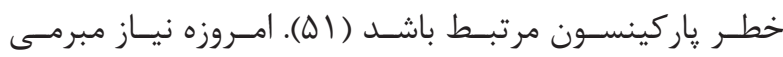

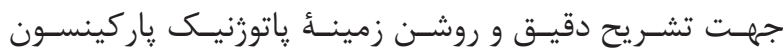

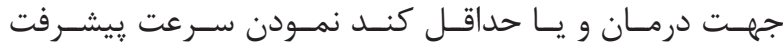

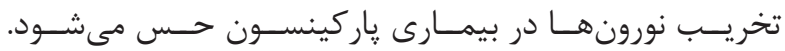

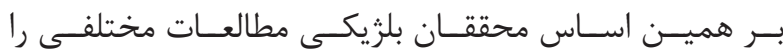

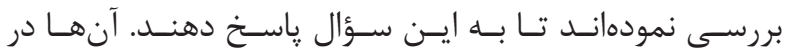

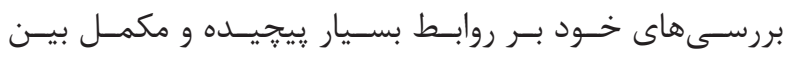

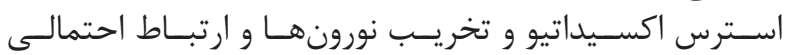

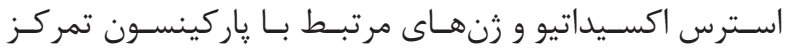

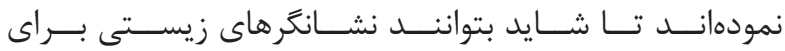

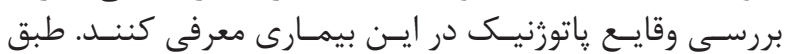

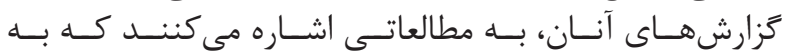

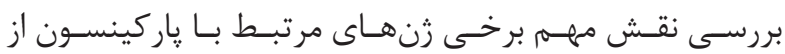

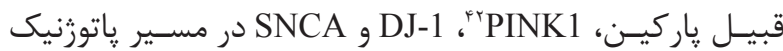

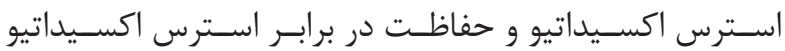

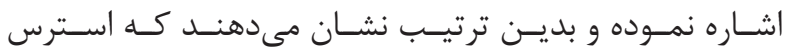

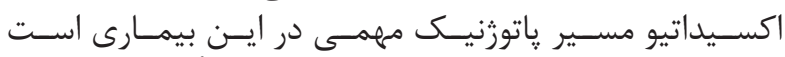

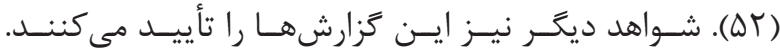

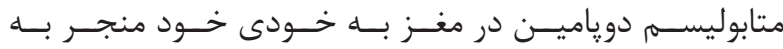

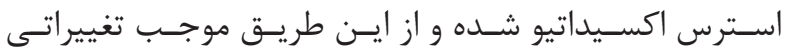

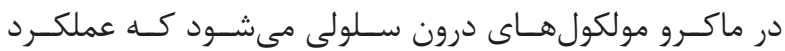

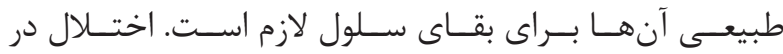

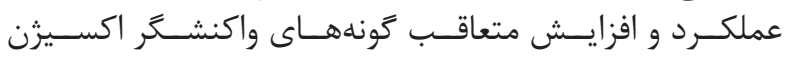

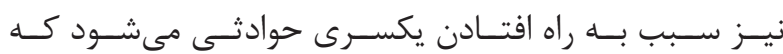

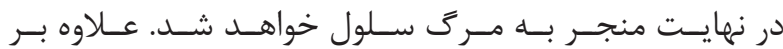

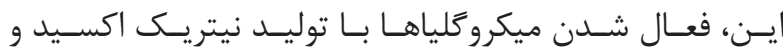

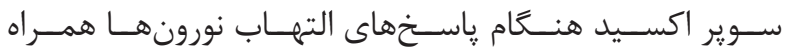

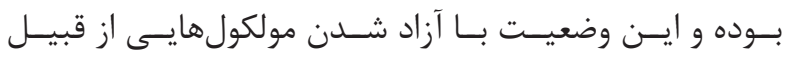

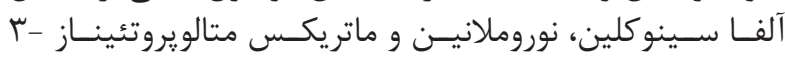

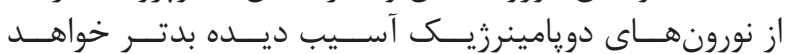

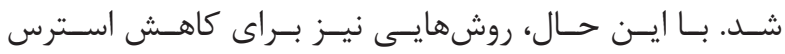

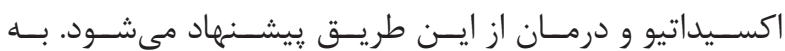

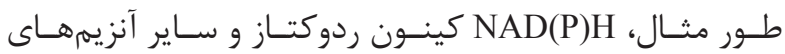

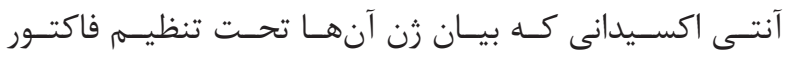

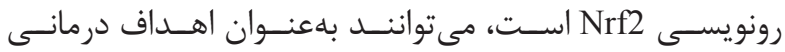

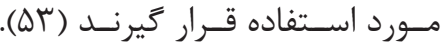

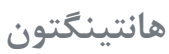

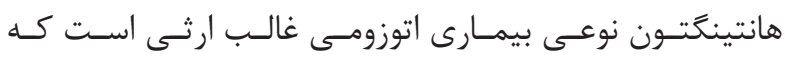

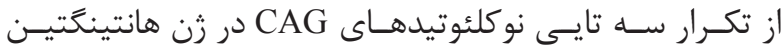

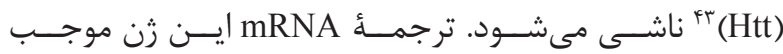

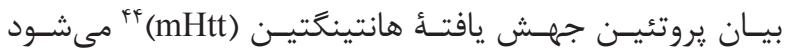
${ }^{44}$ Mutant huntingtin gene

${ }^{45}$ SIRT 
مالتييل اسكلروز

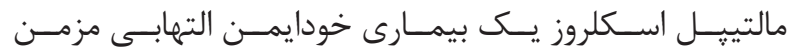

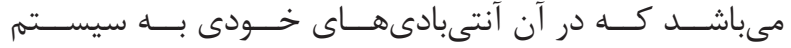

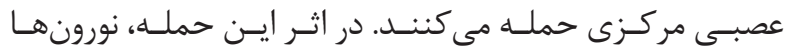

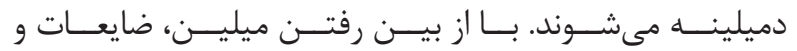

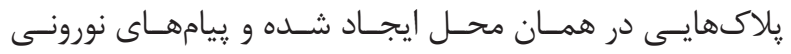

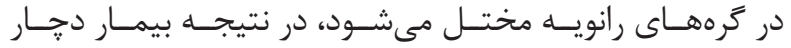

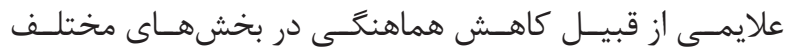

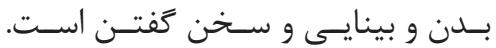

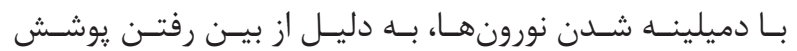

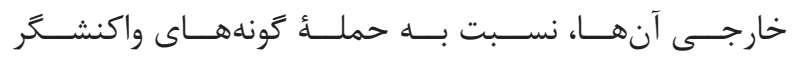

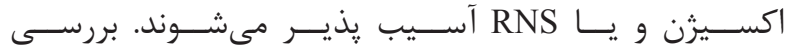

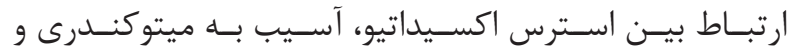

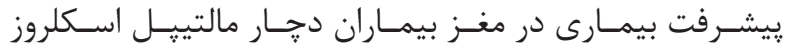

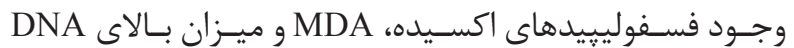

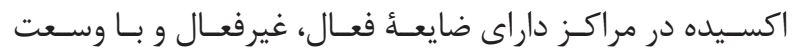

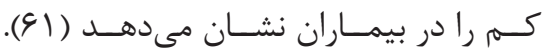

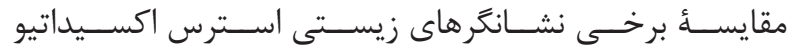

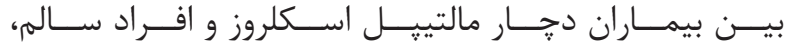

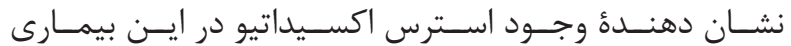

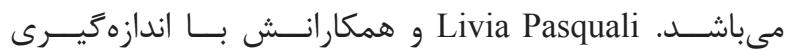

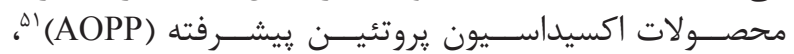

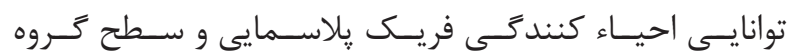

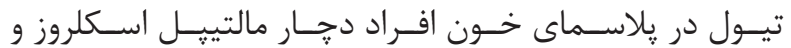

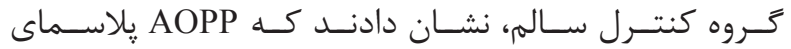

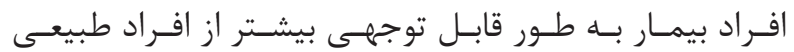

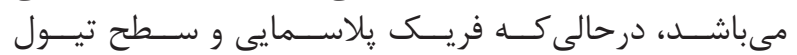

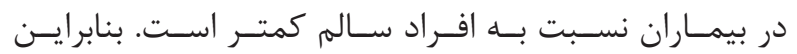

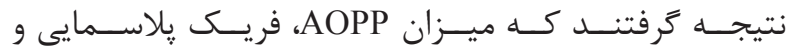

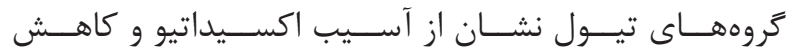

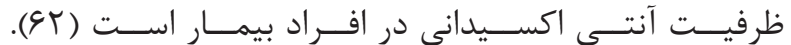

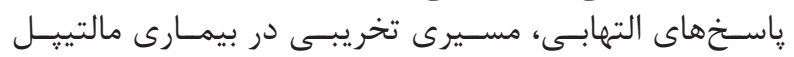

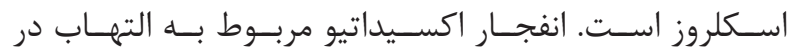

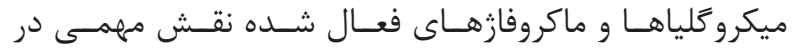

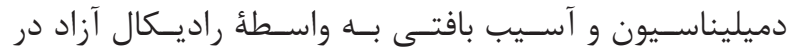

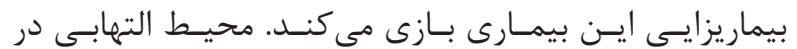

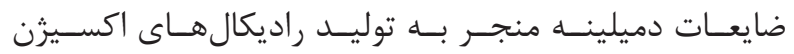

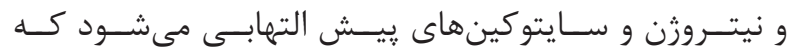

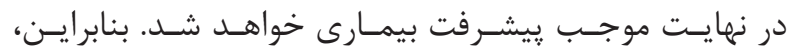

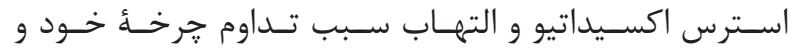

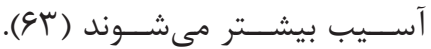

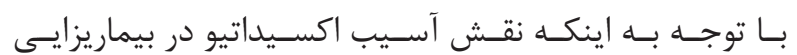

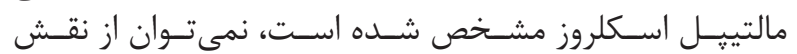

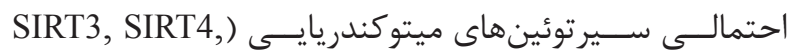

\footnotetext{
${ }^{46}$ Transmitters

${ }^{47}$ PPAR- $\gamma$ co-activator- $1 \alpha$

${ }^{48} \mathrm{~T}$-complex protein 1
}

(SIRT1-7)

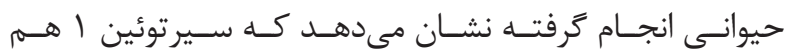

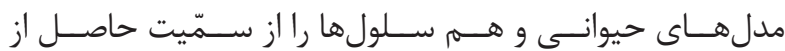

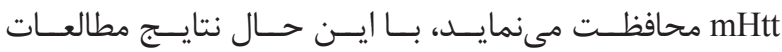

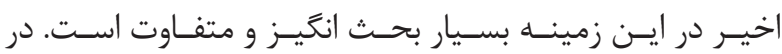

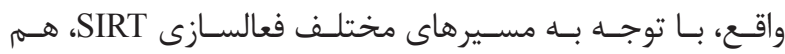

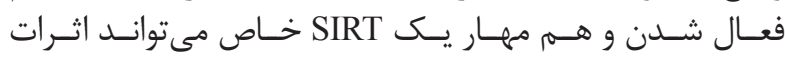

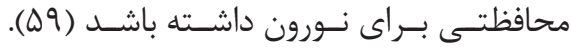

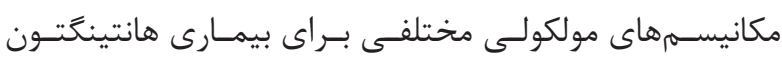

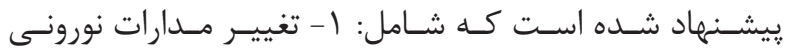

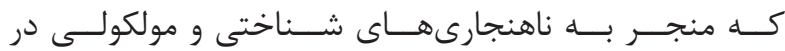

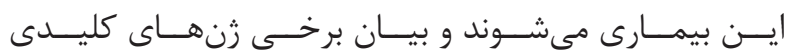

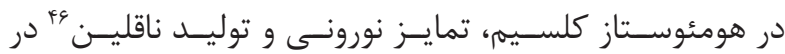

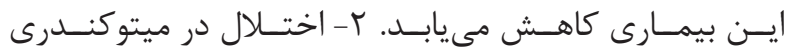

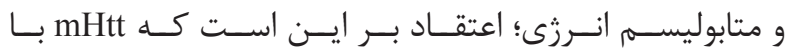

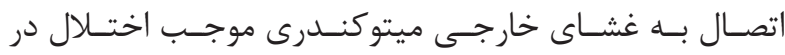

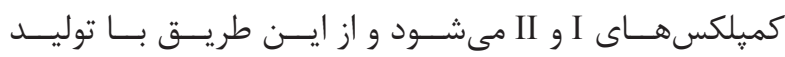

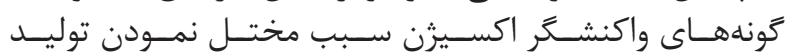

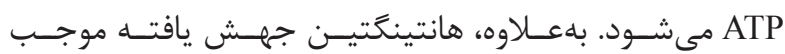

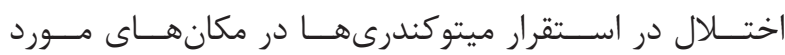

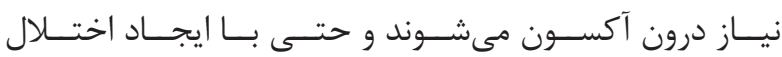

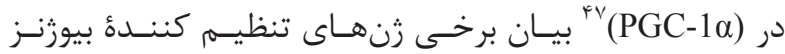

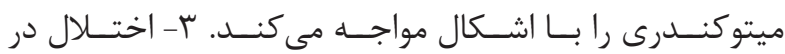

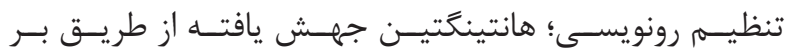

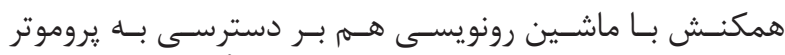

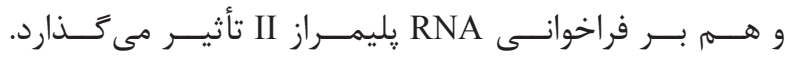

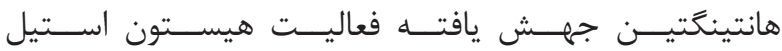

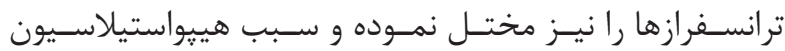

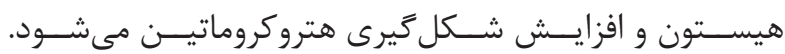

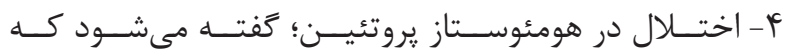

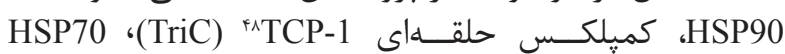

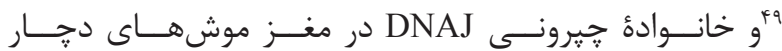

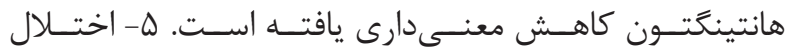

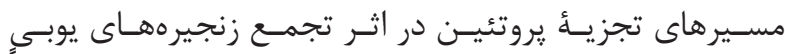

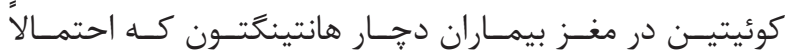

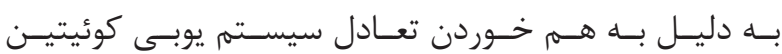

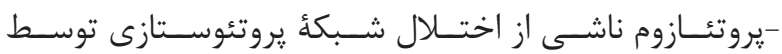

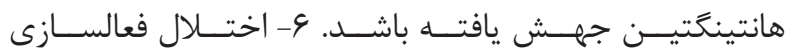

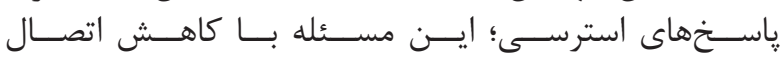

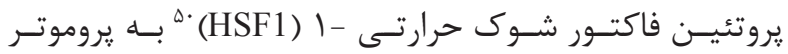

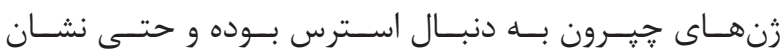

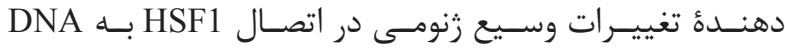

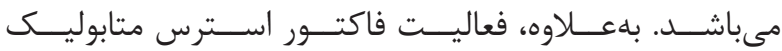

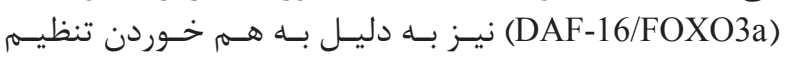

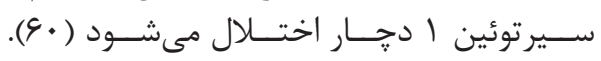

\footnotetext{
${ }^{49} 70$ kilodalton heat shock proteins

${ }^{50}$ Heat shock transcription factor 1

${ }^{51}$ Advanced oxidation protein products
} 


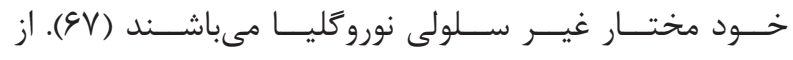

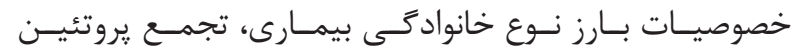

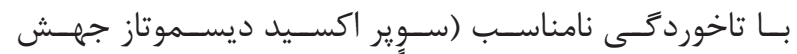

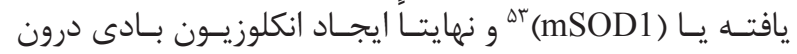

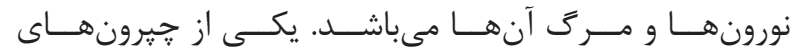

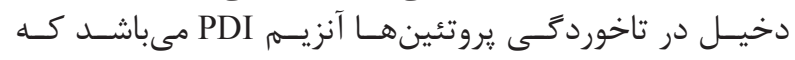

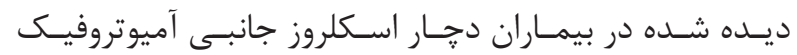

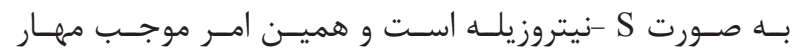

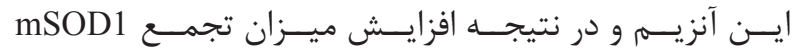

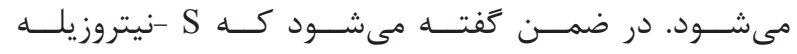

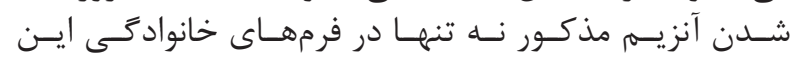

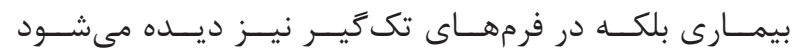

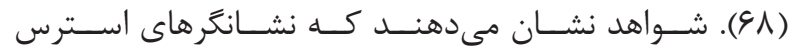

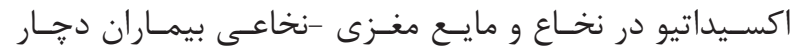

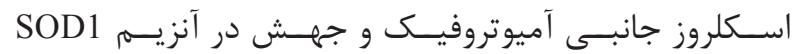

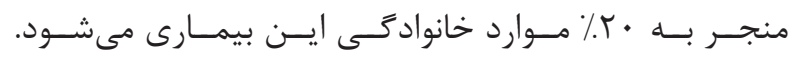

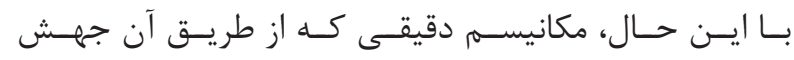

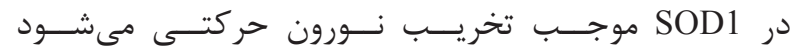

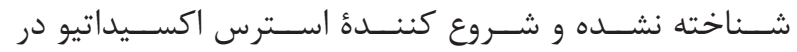

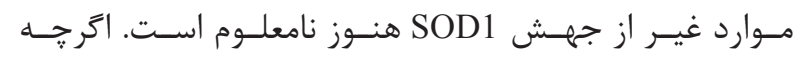

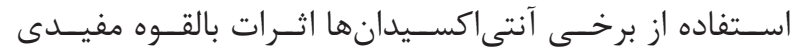

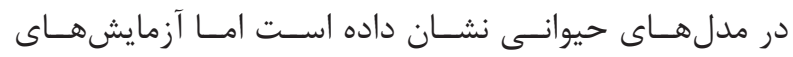

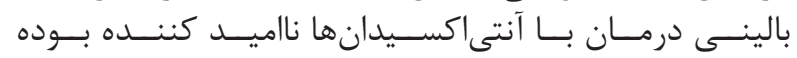

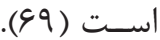

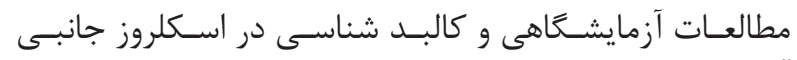

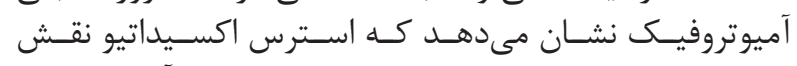

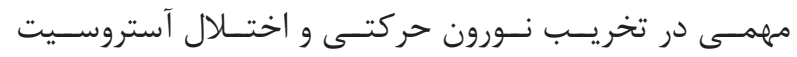

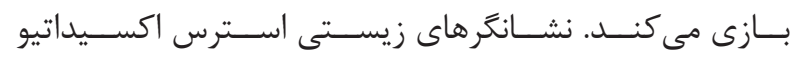

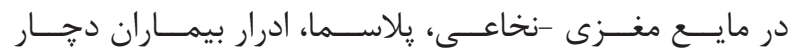

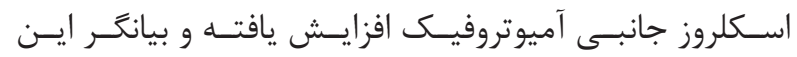

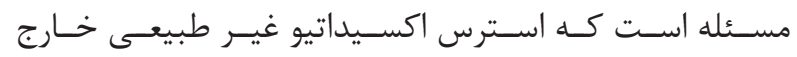

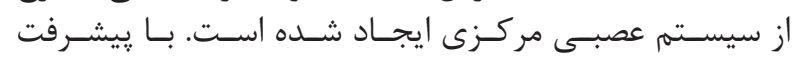

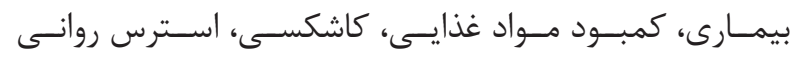

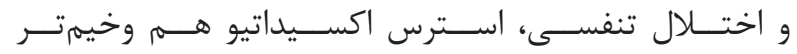

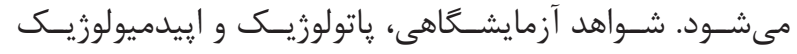

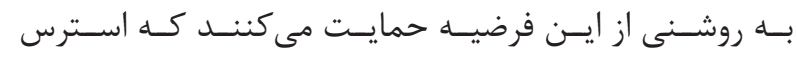

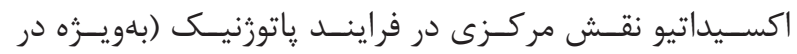

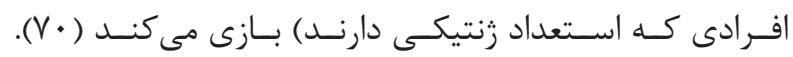

\section{فريدريك آناكسيا}

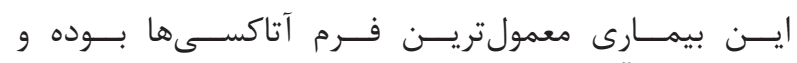

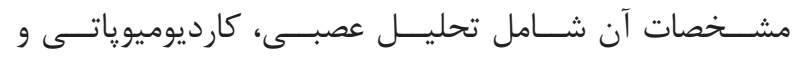

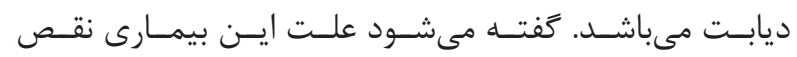

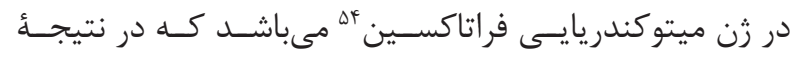

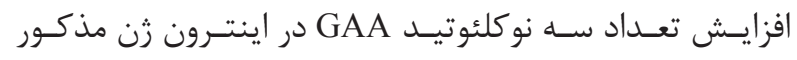

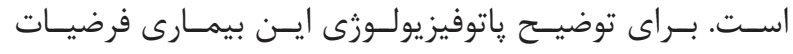

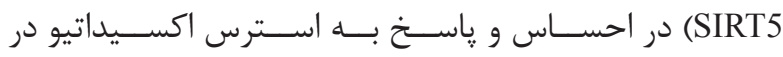

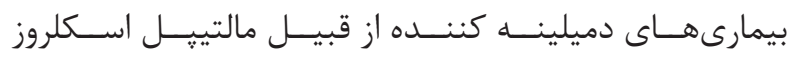

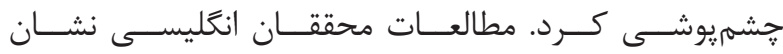

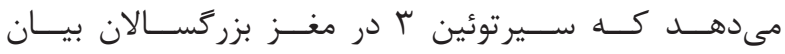

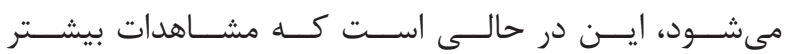

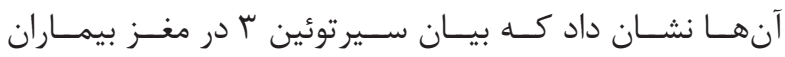

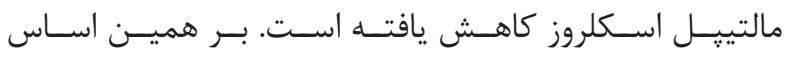

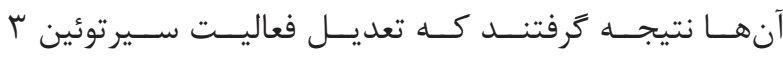

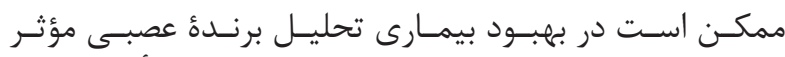

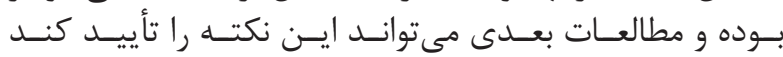

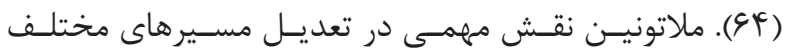

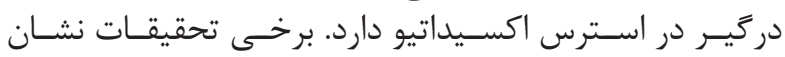

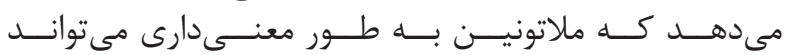

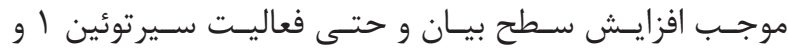

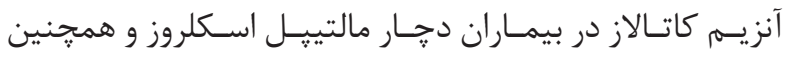

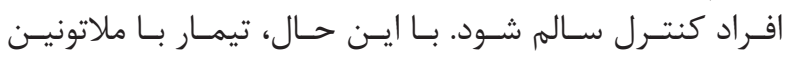

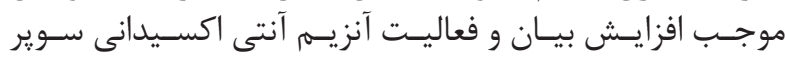

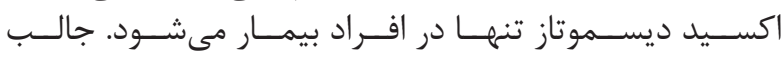

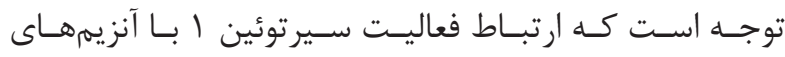

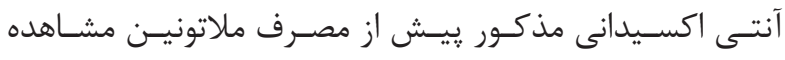

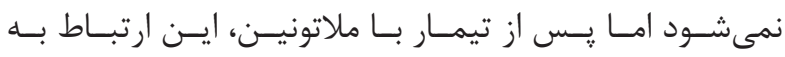

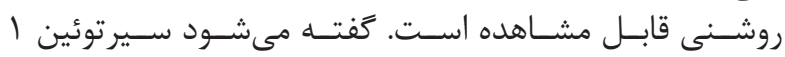

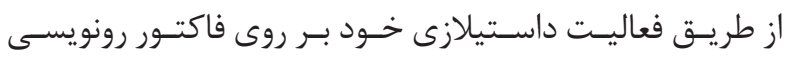

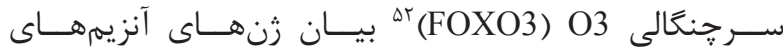

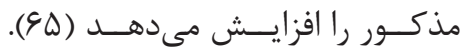

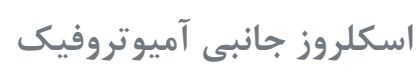

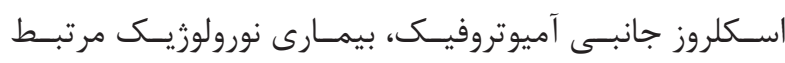

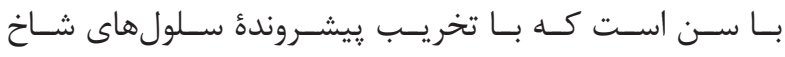

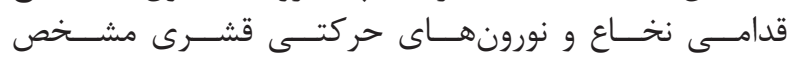

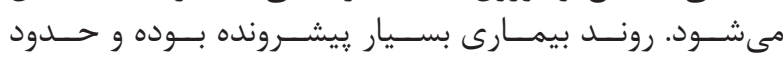

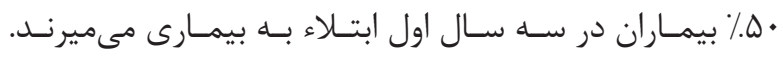

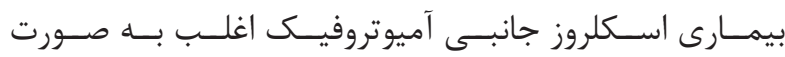

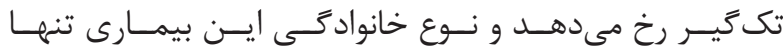

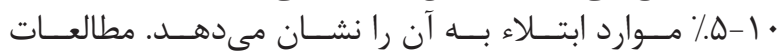

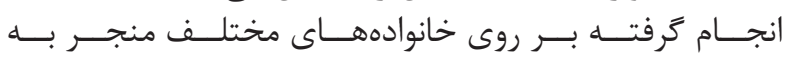

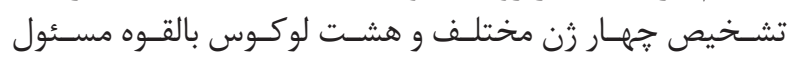

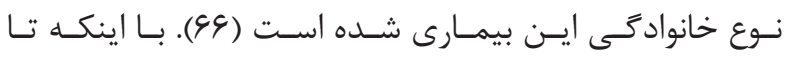

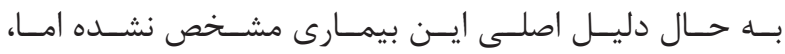

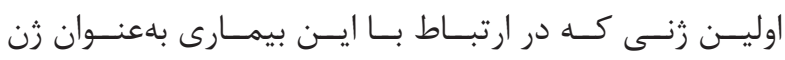

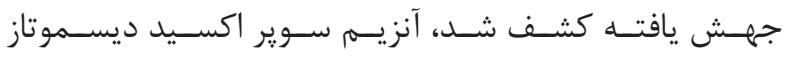

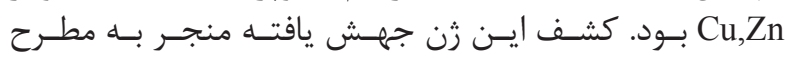

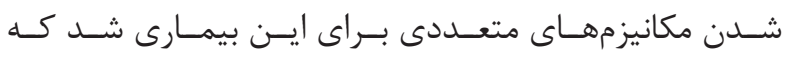

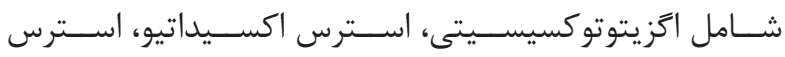

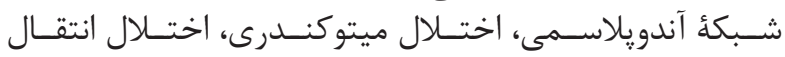

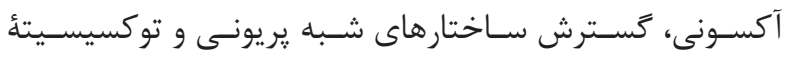

${ }^{52}$ Forkhead box $\mathrm{O} 3$

${ }^{53}$ Mutated form of $\mathrm{Cu}, \mathrm{Zn}$-superoxide dismutase

${ }^{54}$ Frataxin 


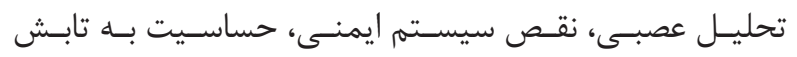

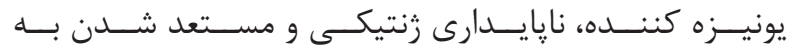

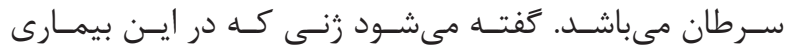

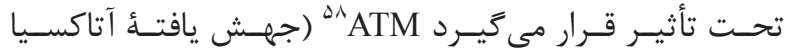

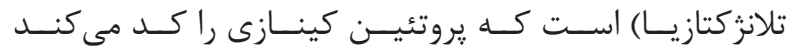

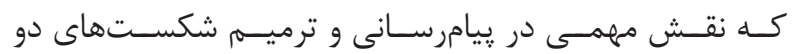

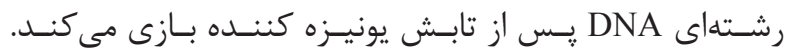

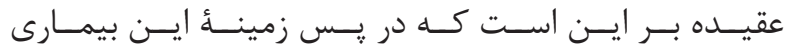

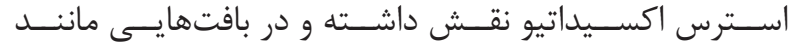

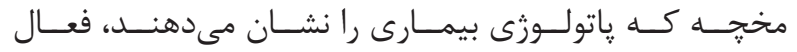

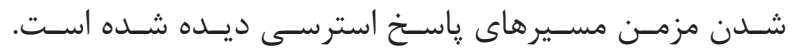

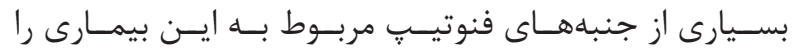

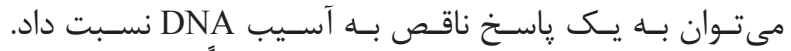

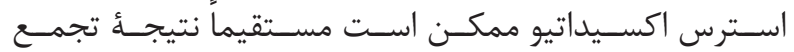

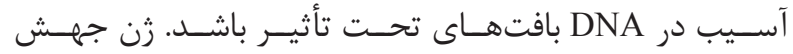

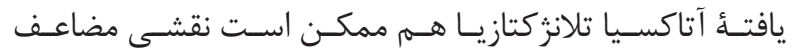

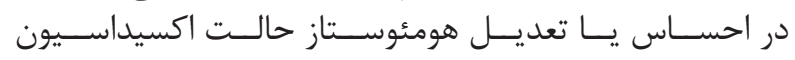
-احيـاء داشـته باشـد (VD)

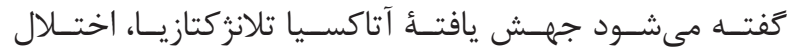

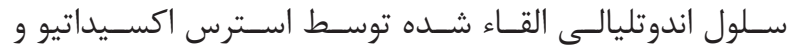

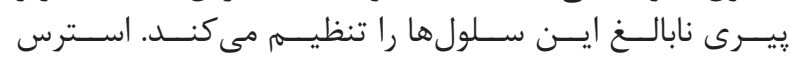

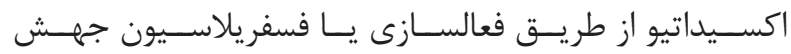

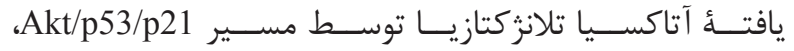

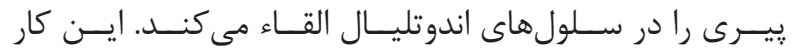

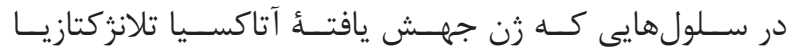

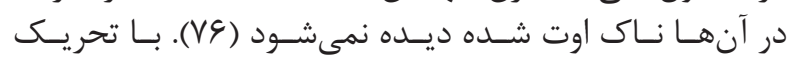

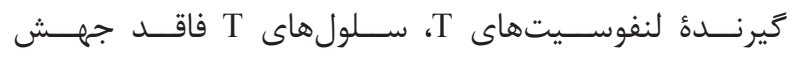

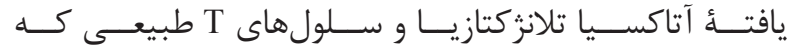

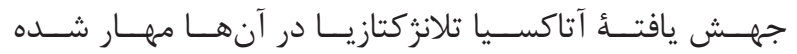

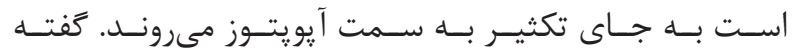

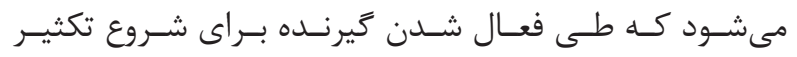

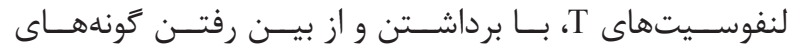

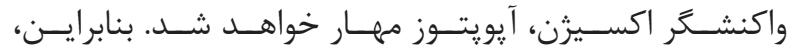

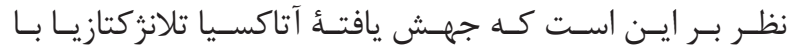

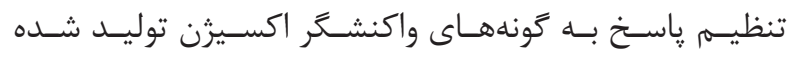

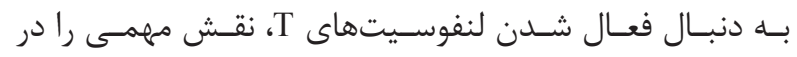

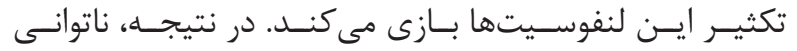

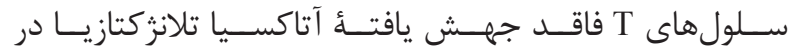

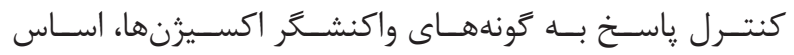

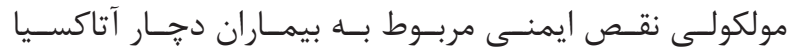

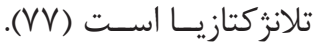

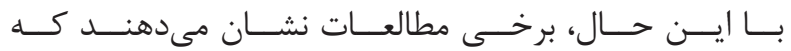

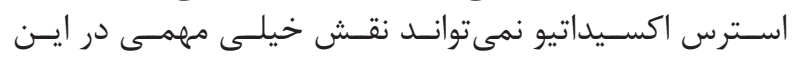

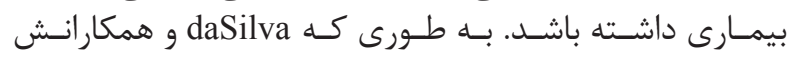

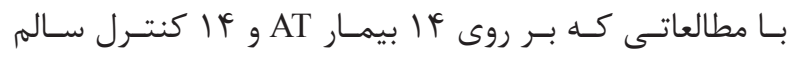

\footnotetext{
${ }^{55}$ Strategies

${ }^{56}$ Polyunsaturated fatty acids
}

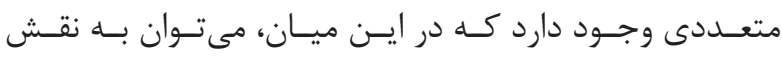

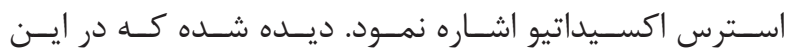

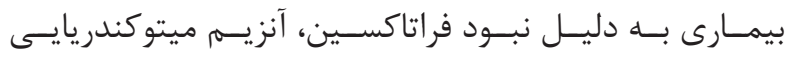

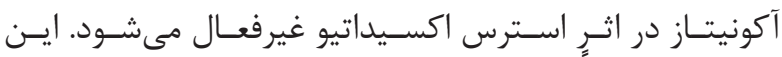

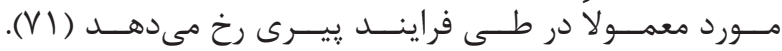

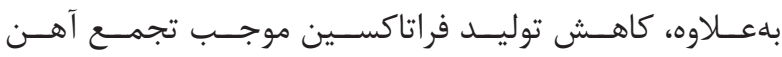

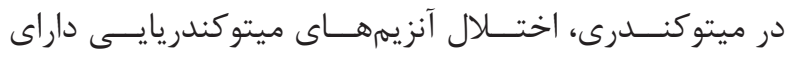
Fe-S

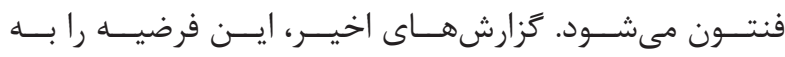

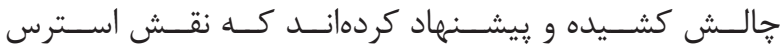

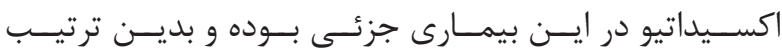

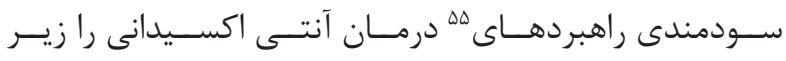

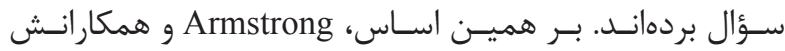

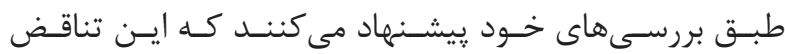

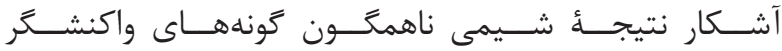

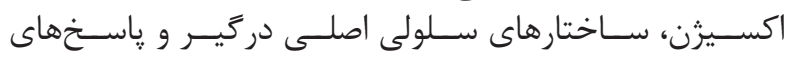

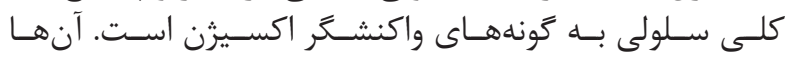

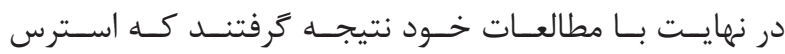

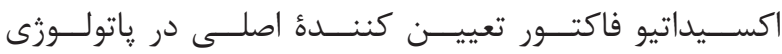

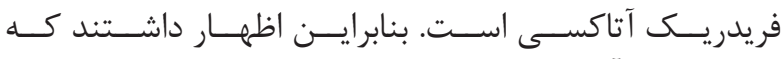

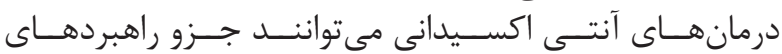

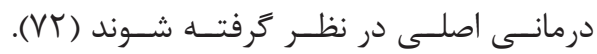

Rosella

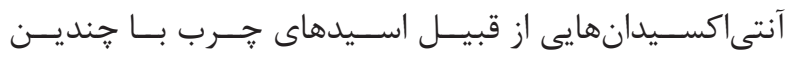

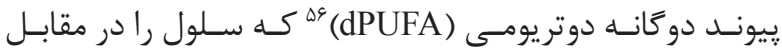

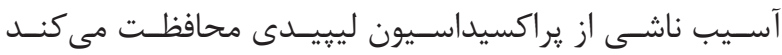

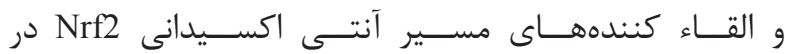

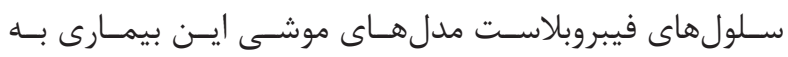

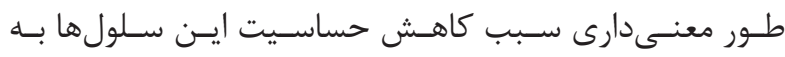

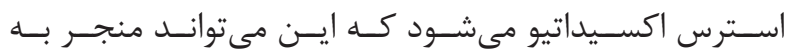

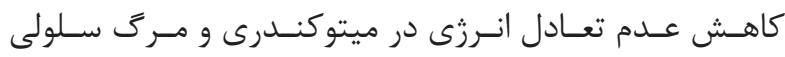

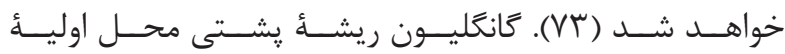

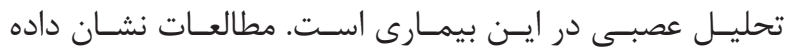

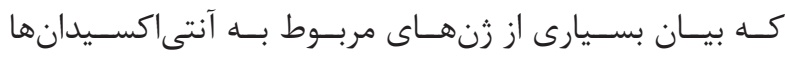

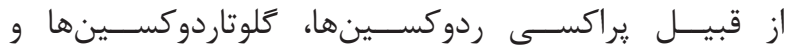

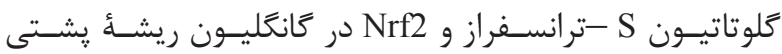

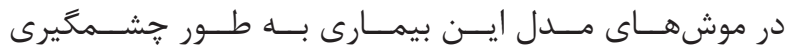

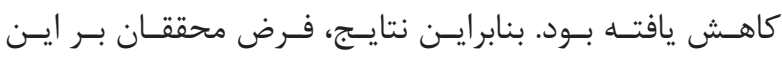

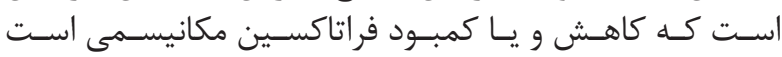

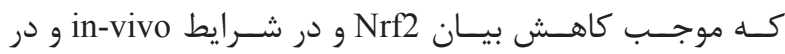

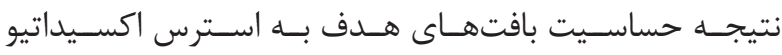

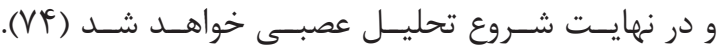
آتاكسيا تلانز كتازيا

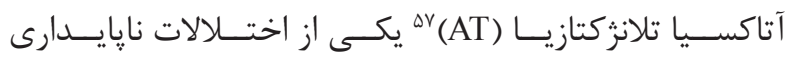

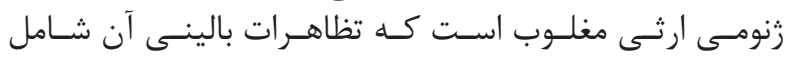

\footnotetext{
${ }^{57}$ Ataxia telangiectasia

${ }^{58}$ Ataxia telangiectasia mutated
} 


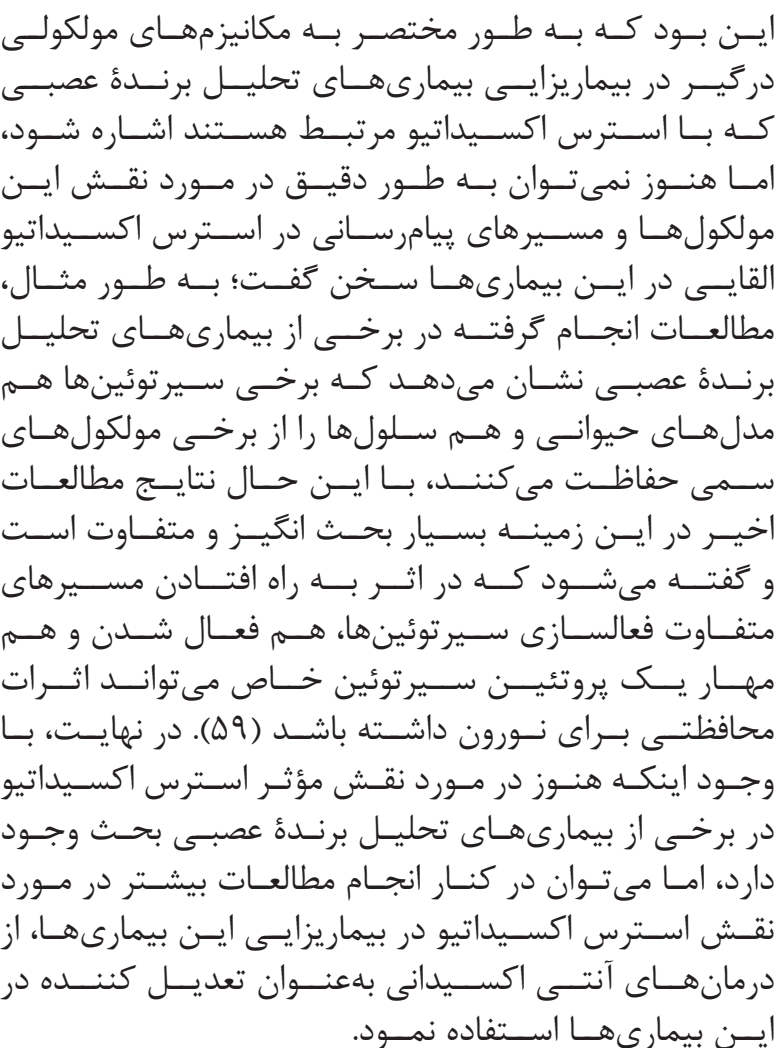

1. Wallace DC. A mitochondrial paradigm of metabolic and degenerative diseases, aging, and cancer: a dawn for evolutionary medicine. Annu Rev Genet. 2005; 39: 359.

2. Rivas -Arancibia S, Gallegos-Ríos C, Briseño DF, Rodríguez-Martínez E, Ferreira-Garcidueñas E, Navarro $\mathrm{L}$, et al. Oxidative stress and neurodegenerative disease: INTECH. 2011; 14(1): 32-8.

3. Melo A, Monteiro L, Lima RM, de Oliveira DM, de Cerqueira MD, El-Bachá RS. Oxidative stress in neurodegenerative diseases: mechanisms and therapeutic perspectives. Oxidative Medicine and Cellular Longevity. 2011; 2011: 1-14.

4. Yun H-Y, Dawson VL, Dawson TM. Neurobiology of nitric oxide. Crit Rev Neurobiol. 1996; 10(3-4): 291-316.

5. Butterfield DA, Castegna A, Lauderback CM, Drake J. Evidence that amyloid beta-peptide-induced lipid peroxidation and its sequelae in Alzheimer's disease brain contribute to neuronal death. Neurobiol Aging. 2002; 23(5): 655-64.

6. Floyd RA, Carney JM. Free radical damage to protein and DNA: mechanisms involved and relevant observations on brain undergoing oxidative stress. Ann Neurol. 1992; 32(S1): S22-S7.

7. Pan X-d, Zhu Y-g, Lin N, Zhang J, Ye Q-y, Huang ${ }^{59}$ Methyl glyoxylate

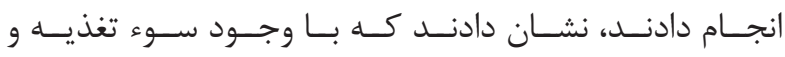

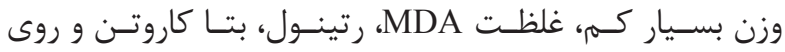

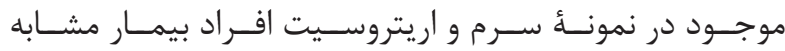

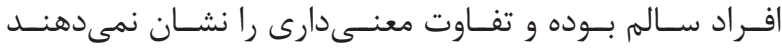

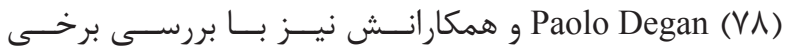

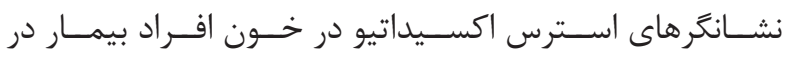

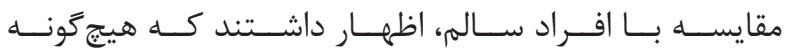

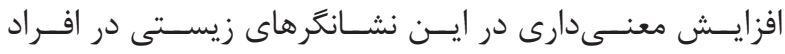

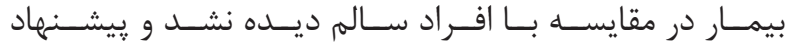

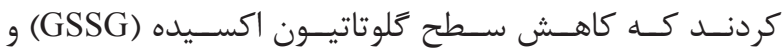

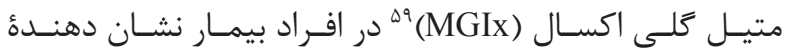

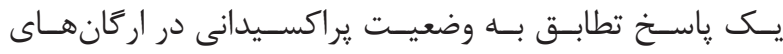

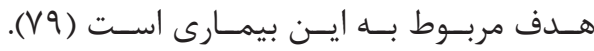
نتيجه

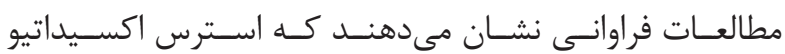

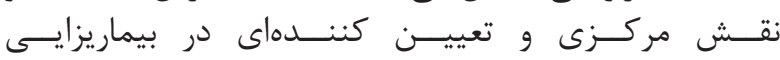

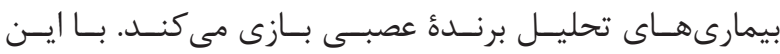

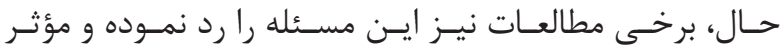

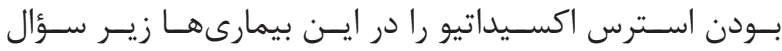

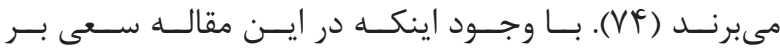

منابع

H-p, et al. Microglial phagocytosis induced by fibrillar $\beta$-amyloid is attenuated by oligomeric $\beta$-amyloid: implications for Alzheimer's disease. Molecular Neurodegeneration. 2011; 6(1): 1-18.

8. Sevcsik E, Trexler AJ, Dunn JM, Rhoades E. Allostery in a disordered protein: oxidative modifications to $\alpha$-synuclein act distally to regulate membrane binding. J Am Chem Soc. 2011; 133(18): 7152-8.

9. Lee J, Kosaras B, Del Signore SJ, Cormier K, McKee A, Ratan RR, et al. Modulation of lipid peroxidation and mitochondrial function improves neuropathology in Huntington's disease mice. Acta Neuropathol. 2011; 121(4): 487-98.

10. Zhao W, Varghese M, Yemul S, Pan Y, Cheng A, Marano $\mathrm{P}$, et al. Peroxisome proliferator activator receptor gamma coactivator-1 alpha (PGC-1 $1 \hat{\mathrm{I}} \pm$ ) improves motor performance and survival in a mouse model of amyotrophic lateral sclerosis. Mol Neurodegener. 2011; 6(1): $1-8$.

11. Witherick J, Wilkins A, Scolding N, Kemp K. Mechanisms of oxidative damage in multiple sclerosis and a cell therapy approach to treatment. Autoimmune diseases. 2010; 2011: doi: 10.4061/2011/164608.

12. Floyd RA, Towner RA, He T, Hensley K, Maples 


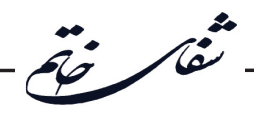

KR. Translational research involving oxidative stress and diseases of aging. Free Radic Biol Med. 2011; 51(5): 931-41.

13. Doroshow J, Hochstein P. Redox cycling and the mechanism of action of antibiotics in neoplastic diseases. Pathology of oxygen: Academic Press. New York: 1982. p. 245-59.

14. Krumova K, Cosa G. Overview of Reactive Oxygen Species. 2016; 1(1): 1-21.

15. Jahanbazi Jahan-Abad, Ali, Parastoo Morteza-zadeh, Sajad Sahab Negah, Ali Gorji. Curcumin attenuates harmful effects of arsenic on neural stem/progenitor cells. AJP. .2017: 1-11.

16. Searing DA, Rabinovitch N. Environmental pollution and lung effects in children. Curr Opin Pediatr. 2011; 23(3): 314-8.

17. Zawia NH, Lahiri DK, Cardozo-Pelaez F. Epigenetics, oxidative stress, and Alzheimer disease. Free Radic Biol Med. 2009; 46(9): 1241-9.

18. Creţu D-I, Sovrea A, Ignat R, Filip A, Bidian C, Creţu A. Morpho-pathological and physiological changes of the brain and liver after ozone exposure. Curr Opin Pediatr. 2010; 51(4): 701-6.

19. Massey V, Schopfer L, Nishino T. Differences in protein structure of xanthine dehydrogenase and xanthine oxidase revealed by reconstitution with flavin active site probes. Free Radical Bio Med. 1989; 264(18): 10567-73.

20. Turrens JF. Mitochondrial formation of reactive oxygen species. The Journal of physiology. 2003; 552(2): 335-44.

21. Al Ghouleh I, Khoo NK, Knaus UG, Griendling KK, Touyz RM, Thannickal VJ, et al. Oxidases and peroxidases in cardiovascular and lung disease: new concepts in reactive oxygen species signaling. Free Radic Biol Med. 2011; 51(7): 1271-88.

22. Brand MD. Mitochondrial generation of superoxide and hydrogen peroxide as the source of mitochondrial redox signaling. Free Radic Biol Med. 2016. doi: $10.1016 /$ j.

23. Araki T, Kumagai T, Tanaka K, Matsubara M, Kato $\mathrm{H}$, Itoyama $\mathrm{Y}$, et al. Neuroprotective effect of riluzole in MPTP-treated mice. Brain Research. 2001; 918(1): 176-81.

24. Jiang Z, Hu Z, Zeng L, Lu W, Zhang H, Li T, et al.
The role of the Golgi apparatus in oxidative stress: is this organelle less significant than mitochondria? Free Radic Biol Med. 2011; 50(8): 907-17.

25. Frei B. Reactive oxygen species and antioxidant vitamins: mechanisms of action. The Am J Med. 1994; 97(3): S5-S13.

26. Ferrari CKB. Free radicals, lipid peroxidation and antioxidants in apoptosis: implications in cancer, cardiovascular and neurological diseases. Biologia Bratislava. 2000; 55(6): 581-90.

27. Ian H. Enols and enolates- the michael additions. University of Calgary. http://en.wikipedia.org/wiki/ Michael_addition\#cite_note-0 . 2008.

28. Keller JN, Pang Z, Geddes JW, Begley JG, Germeyer A, Waeg G, et al. Impairment of glucose and glutamate transport and induction of mitochondrial oxidative stress and dysfunction in synaptosomes by amyloid $\beta$-peptide: Role of the lipid peroxidation product 4-hydroxynonenal. J Neurochem. 1997; 69(1): 273-84.

29. Ermak G, Davies KJ. Calcium and oxidative stress: from cell signaling to cell death. Mol Immunol. 2002; 38(10): 713-21.

30. Mattson MP. Excitotoxic and excitoprotective mechanisms. NeuroMolecular Med. 2003; 3(2): 65-94.

31. Halima SB, Mishra S, Raja KMP, Willem M, Baici A, Simons K, et al. Specific inhibition of $\beta$-secretase processing of the Alzheimer disease amyloid precursor protein. Cell Rep. 2016; 14(9): 2127-41.

32. Ahmadi J, Jahanbazi Jahan Abad A, Barahimi A, Atashi A. Introduction of Long Non-Coding RNAs as Novel Biomarkers in Central Nervous System Disorders. Neurosci J Shefaye Khatam. 2015 :98-112.

33. Greilberger J, Koidl C, Greilberger M, Lamprecht M, Schroecksnadel K, Leblhuber F, et al. Malondialdehyde, carbonyl proteins and albumin-disulphide as useful oxidative markers in mild cognitive impairment and Alzheimer's disease. Free Radical Res. 2008; 42(7): 633-8.

34. Sultana R, Perluigi M, Butterfield DA. Lipid peroxidation triggers neurodegeneration: a redox proteomics view into the Alzheimer disease brain. Free Radic Biol Med. 2013; 62: 157-69.

35. Turunc Bayrakdar E, Uyanikgil Y, Kanit L, Koylu E, Yalcin A. Nicotinamide treatment reduces the levels of oxidative stress, apoptosis, and PARP-1 activity in A $\beta$ (1-42)-induced rat model of Alzheimer's disease. Free 
Radical Res. 2014; 48(2): 146-58.

36. Chiarugi A. Intrinsic mechanisms of poly (ADPribose) neurotoxicity: three hypotheses. Neurotoxicology. 2005; 26(5): 847-55.

37. Strosznajder JB, Czapski GA, Adamczyk A, Strosznajder RP. Poly (ADP-ribose) polymerase-1 in amyloid beta toxicity and Alzheimer's disease. Mol Neurobiol. 2012; 46(1): 78-84.

38. Matsumura A, Emoto MC, Suzuki S, Iwahara N, Hisahara S, Kawamata J, et al. Evaluation of oxidative stress in the brain of a transgenic mouse model of Alzheimer's disease by in vivo electron paramagnetic resonance imaging. Free Radic Biol Med. 2015; 85: $165-73$.

39. Wang S-w, Yang S-g, Liu W, Zhang Y-x, Xu P-x, Wang $T$, et al. Alpha-tocopherol quinine ameliorates spatial memory deficits by reducing beta-amyloid oligomers, neuroinflammation and oxidative stress in transgenic mice with Alzheimer's disease. Behav Brain Res. 2016; 296: 109-17.

40. Hritcu L, Stefan M, Brandsch R, Mihasan M. Enhanced behavioral response by decreasing brain oxidative stress to 6-hydroxy-1-nicotine in Alzheimer's disease rat model. Neurosci Lett. 2015; 591: 41-7.

41. Forestier A, Douki T, De Rosa V, Béal D, Rachidi W. Combination of $\mathrm{A} \beta$ secretion and oxidative stress in an alzheimer-like cell line leads to the over-expression of the nucleotide excision repair proteins ddb2 and XPC. Int J Mol Sci. 2015; 16(8): 17422-44.

42. Babaei Abraki S, Chavoshi-Nezhad S. Mitochondrial defects and oxidative stress in Alzheimer disease. Shefaye Khatam. 2014; 2(1): 85-94.

43. Babaei Abraki S, Chavoshi-Nezhad S. Alzheimer's disease: The effect of nrf2 signaling pathway on cell death caused by oxidative stress. Shefaye Khatam. 2015; 3(1): 145-56.

44. Mota SI, Costa RO, Ferreira IL, Santana I, Caldeira GL, Padovano $\mathrm{C}$, et al. Oxidative stress involving changes in Nrf2 and ER stress in early stages of Alzheimer's disease. Biochim Biophys Acta. 2015; 1852(7): 1428-41.

45. Hernández-Zimbrón L, Rivas-Arancibia S. Oxidative stress caused by ozone exposure induces $\beta$-amyloid 1-42 overproduction and mitochondrial accumulation by activating the amyloidogenic pathway. Neuroscience. 2015; 304: 340-8.

46. Thomas B, Beal MF. Parkinson's disease. Hum Mol
Genet. 2007; 16(2): R183-94.

47. Moszczynska A, Yamamoto BK. Methamphetamine oxidatively damages parkin and decreases the activity of $26 \mathrm{~S}$ proteasome in vivo. J Neurochem. 2011; 116(6): 1005-17.

48. Siddiqui A, Rane A, Rajagopalan S, Chinta SJ, Andersen JK. Detrimental effects of oxidative losses in parkin activity in a model of sporadic Parkinson's disease are attenuated by restoration of PGC1alpha. Neurobiol Dis. 2016; 93: 115-20.

49. Tsang AH, Lee Y-I, Ko HS, Savitt JM, Pletnikova O, Troncoso JC, et al. S-nitrosylation of XIAP compromises neuronal survival in Parkinson's disease. Proc Natl Acad Sci U S A. 2009; 106(12): 4900-5.

50. Ryan SD, Dolatabadi N, Chan SF, Zhang X, Akhtar MW, Parker J, et al. Isogenic human iPSC Parkinson's model shows nitrosative stress-induced dysfunction in MEF2-PGC1 $\alpha$ transcription. Cell. 2013; 155(6): 1351-64.

51. Tsai K-L, Cheng Y-Y, Leu H-B, Lee Y-Y, Chen T-J, Liu D-H, et al. Investigating the role of sirt1-modulated oxidative stress in relation to benign paroxysmal positional vertigo and Parkinson's disease. N Neurobiol Aging. 2015; 36(9): 2607-16.

52. Varçin M, Bentea E, Michotte Y, Sarre S. Oxidative stress in genetic mouse models of Parkinson's disease. Oxid Med Cell Longev. 2012; 2012. doi: $10.1155 / 2012 / 624925$.

53. Hwang O. Role of oxidative stress in Parkinson's disease. Exp Neurobiol. 2013; 22(1): 11-7.

54. Keum JW, Shin A, Gillis T, Mysore JS, Elneel KA, Lucente D, et al. The HTT CAG-expansion mutation determines age at death but not disease duration in huntington disease. Am J Hum Genet. 2016; 98(2): 287-98.

55. Zuccato C, Valenza M, Cattaneo E. Molecular mechanisms and potential therapeutical targets in Huntington's disease. Physiol Rev. 2010; 90(3): 905-81.

56. Berggren KL, Chen J, Fox J, Miller J, Dodds L, Dugas B, et al. Neonatal iron supplementation potentiates oxidative stress, energetic dysfunction and neurodegeneration in the R6/2 mouse model of Huntington's disease. Redox Biol. 2015; 4: 363-74.

57. Ju T-C, Chen H-M, Chen Y-C, Chang C-P, Chang C, Chern Y. AMPK- $\alpha 1$ functions downstream of oxidative stress to mediate neuronal atrophy in Huntington's disease. Biochim Biophys Acta. 2014; 1842(9): 1668-80. 


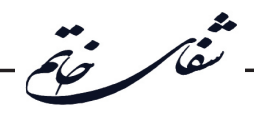

58. Ribeiro M, Rosenstock TR, Oliveira AM, Oliveira CR, Rego AC. Insulin and IGF-1 improve mitochondrial function in a PI-3K/Akt-dependent manner and reduce mitochondrial generation of reactive oxygen species in Huntington's disease knock-in striatal cells. Free Rad Biol Med. 2014; 74: 129-44.

59. Naia L, Rego AC. Sirtuins: double players in Huntington's disease. Biochim Biophys Acta. 2015; 1852(10): 2183-94.

60. Labbadia J, Morimoto RI. Huntington's disease: underlying molecular mechanisms and emerging concepts. Trends Biochem Sci. 2013; 38(8): 378-85.

61. Haider L, Fischer MT, Frischer JM, Bauer J, Höftberger R, Botond $\mathrm{G}$, et al. Oxidative damage in multiple sclerosis lesions. Brain. 2011; 134(7): 1914-24.

62. Pasquali L, Pecori C, Lucchesi C, LoGerfo A, Iudice A, Siciliano G, et al. Plasmatic oxidative stress biomarkers in multiple sclerosis: relation with clinical and demographic characteristics. Clin biochem. 2015; 48(1): $19-23$.

63. Ortiz GG, Pacheco-Moisés FP, Bitzer-Quintero OK, Ramírez-Anguiano AC, Flores-Alvarado LJ, RamírezRamírez V, et al. Immunology and oxidative stress in multiple sclerosis: clinical and basic approach. Clin Dev Immunol. 2013; 2013. doi: 10.1155/2013/708659.

64. Rice C, Sun M, Kemp K, Gray E, Wilkins A, Scolding N. Mitochondrial sirtuins-a new therapeutic target for repair and protection in multiple sclerosis. EUR J Neurosci. 2012; 35(12): 1887-93.

65. Emamgholipour S, Hossein-nezhad A, Sahraian MA, Askarisadr F, Ansari M. Evidence for possible role of melatonin in reducing oxidative stress in multiple sclerosis through its effect on SIRT1 and antioxidant enzymes. Life Sci. 2016; 145: 34-41.

66. Kiernan MC, Vucic S, Cheah BC, Turner MR, Eisen A, Hardiman O, et al. Amyotrophic lateral sclerosis. The Lancet. 2011; 377(9769): 942-55.

67. Hayashi Y, Homma K, Ichijo H. SOD1 in neurotoxicity and its controversial roles in SOD1 mutation-negative ALS. Adv Biol Regul. 2016; 60: 95-104.

68. Jeon GS, Nakamura T, Lee J-S, Choi W-J, Ahn S-W, Lee K-W, et al. Potential effect of S-nitrosylated protein disulfide isomerase on mutant SOD1 aggregation and neuronal cell death in amyotrophic lateral sclerosis. Mol Neurobiol. 2014; 49(2): 796-807.
69. Barber SC, Shaw PJ. Oxidative stress in ALS: key role in motor neuron injury and therapeutic target. Free Radic Biol Med. 2010; 48(5): 629-41.

70. D’Amico E, Factor-Litvak P, Santella RM, Mitsumoto H. Clinical perspective on oxidative stress in sporadic amyotrophic lateral sclerosis. Free Radic Biol Med. 2013; 65: 509-27.

71. Llorens JV, Navarro JA, Martínez-Sebastián MJ, Baylies MK, Schneuwly S, Botella JA, et al. Causative role of oxidative stress in a Drosophila model of Friedreich ataxia. FASEB J. 2007; 21(2): 333-44.

72. Armstrong JS, Khdour O, Hecht SM. Does oxidative stress contribute to the pathology of Friedreich's ataxia? a radical question. FASEB J. 2010; 24(7): 2152-63.

73. Abeti R, Uzun E, Renganathan I, Honda T, Pook MA, Giunti P. Targeting lipid peroxidation and mitochondrial imbalance in Friedreich's ataxia. Pharmacol Res. 2015; 99: 344-50.

74. Shan Y, Schoenfeld RA, Hayashi G, Napoli E, Akiyama T, Iodi Carstens M, et al. Frataxin deficiency leads to defects in expression of antioxidants and Nrf2 expression in dorsal root ganglia of the Friedreich's ataxia YG8R mouse model. Antioxid Redox Signal. 2013; 19(13): 1481-93.

75. Watters DJ. Oxidative stress in ataxia telangiectasia. Redox Rep. 2003; 8(1): 23-9.

76. Zhan H, Suzuki T, Aizawa K, Miyagawa K, Nagai R. Ataxia telangiectasia mutated (ATM)-mediated DNA damage response in oxidative stress-induced vascular endothelial cell senescence. J Biol Chem. 2010; 285(38): 29662-70.

77. Bagley J, Singh G, Iacomini J. Regulation of oxidative stress responses by ataxia-telangiectasia mutated is required for T cell proliferation. J Immunol. 2007; 178(8): 4757-63.

78. Da Silva R, dos Santos-Valente E, Scomparini FB, Sarni RS, Costa-Carvalho B. The relationship between nutritional status, vitamin A and zinc levels and oxidative stress in patients with ataxia-telangiectasia. Allergol Immunopathol (Madr). 2014; 42(4): 329-35.

79. Degan P, d'Ischia M, Pallardó FV, Zatterale A, Brusco A, Calzone R, et al. Glutathione levels in blood from ataxia telangiectasia patients suggest in vivo adaptive mechanisms to oxidative stress. Clin Biochem. 2007; 40(9): 666-70. 